\title{
Optimal Risk Sharing with Background Risk
}

\author{
Rose-Anne Dana* \\ CEREMADE \\ Université Paris Dauphine \\ Place du Maréchal de Lattre de Tassigny \\ F-75775 Paris Cedex 16, France \\ dana@ceremade.dauphine.fr \\ Marco Scarsini ${ }^{\dagger}$ \\ Dipartimento di Statistica e Matematica Applicata \\ Università di Torino \\ Piazza Arbarello 8 \\ I-10122 Torino, Italy \\ marco.scarsini@unito.it
}

March 29, 2005

\begin{abstract}
This paper examines qualitative properties of efficient insurance contracts in the presence of background risk. In order to get results for all strictly risk averse expected utility maximizers, the concept of "stochastic increasingness" is used. Different assumptions on the stochastic dependence between the insurable and uninsurable risk lead to different qualitative properties of the efficient contracts. The new results obtained under hypotheses of dependent risks are compared to classical results in the absence of background risk or to the case of independent risks. The theory is further generalized to nonexpected utility maximizers.
\end{abstract}

JEL Classification: D52, G22

Keywords: insurance, efficient contracts, incomplete markets, stochastically increasing.

*This author acknowledges the support of the Fondation du Risque, chaire Dauphine-ENSAE-Groupama, "Les particuliers face au risque" and of the ANR project "Croyances"

†The work of this author was partially supported by MIUR-COFIN 


\section{Introduction}

Since the early work of Borch (1962), many authors have considered the problem of the optimal sharing of risk between an insurer and an insured. In particular Arrow (1963, 1970, 1974) showed that if the premium set by a risk neutral insurer depends only on the actuarial value of the policy offered and is fair, then the optimal policy for a risk averse von-Neumann Morgenstern insured is full insurance. If the premium includes a factor loading, then the optimal policy contains full insurance above a deductible. This result plays an important role in the literature since it shows that the insured's decision can be brought down to a one-dimensional problem, the choice of the optimal deductible (or equivalently of the optimal premium). In particular, it allows for comparative static results.

Raviv (1979) reconsidered the problem under a more general set of assumptions: the insurer can be risk averse and the premium is a convex function of the supplied insurance. He showed that if the marginal cost is greater than 1, then the efficient policy of a risk averse von-Neumann Morgenstern insured entails coinsurance above a deductible. Furthermore, if the insurer is risk averse and if the cost of insurance equals the supplied insurance, then the efficient policy is coinsurance. It may also be checked that if the cost is too high the insured takes no insurance.

These results have at least two important implications: first, insurer and insured wealths are comonotone, each being nonincreasing function of the risk (in other words, there is risk sharing). Second, if there are no costs, then there are no deductibles, and, if there are costs, then there are deductibles. Hence costs explain deductibles.

A drawback of this analysis is that it assumes that markets are complete. As already mentioned by Doherty and Schlesinger (1983a,b), Schlesinger and Doherty (1985) and Gollier (1996), first insurers prefer to cover different sources of risk by different contracts and next, some risks such as war, floods, earthquakes, market risks, and human capital are not insurable. Hence, it seems necessary to reconsider insurance problems under the assumption of background risk. Furthermore, the problem of insurance in the presence of background risk arises in the pricing of climatic options (the risk to be insured is a climatic risk and the background risk is the financial risk, see for example Barrieu and El Karoui (2002, 2003)). It also arises when an insured faces a sequence of risks over time and chooses at each date an insurance contract that depends on the forthcoming risk and on her history.

It is well-known that the presence of background risk in wealth has an effect on the demand for other risks. Several authors have considered different risk postures of decision makers in the presence of background risk, among them Ross (1981), Kihlstrom et al. (1981), Nachman (1982), Pratt and Zeckhauser (1987), Jewitt (1987), Kimball (1993), Gollier and Pratt (1996), Eeckhoudt et al. (1996), Finkelshtain et al. (1999), Franke et al. (2004). An extended treatment of background risk and relevant references can be found in Gollier (2001).

Insurance with background risk has been considered in various settings, since the early work of Doherty and Schlesinger (1983b). Doherty and Schlesinger (1983b) first addressed the problem of insurance demand with background risk in a two-state economy. The problem

has been reconsidered by a number of authors (see the survey paper by Schlesinger (2000) who discussed the case of proportional coinsurance under independent and dependent background 
risk). This trend in the literature has focused for the most part on comparing demand when there is background risk to demand when there is no background risk. Eeckhoudt and Kimball (1992) have considered background risk of loss such that a higher level of insurable risk implies a less risky distribution of the background risk loss in the sense of third degree stochastic dominance. In the setting of an exchange economy where agents have HARA utilities, Franke et al. (1998) studied the effect of introducing an independent background risk.

Gollier (1996) first examined the problem of efficient contracts when there is background risk under the assumptions that the insurer is risk neutral, and that the premium is a function of the expected indemnity, with marginal increase greater than 1. Assuming two dependent sources of risks such that the uninsurable risk has zero conditional expectation given any value of the insurable risk, he showed that if a higher level of insurable risk implies a more risky distribution of the background risk, then the deductible rule does not apply anymore. He showed also that if the insured is prudent, then the optimal insurance contract entails a disappearing deductible. Efficient contracts when there is background risk were also considered by Mahul (2000) and Vercammen (2001) in slightly different models, in which the risks are independent, but the loss in revenue is not additive.

This paper shows that the shape of the optimal insurance contract crucially depends on the type of dependence among the insurable and noninsurable risks.

The optimal contract results, to be stated in the sequel, concern a large class of decision makers, and entail no parametric assumptions of the distribution of risks. All strictly risk averse expected utility maximizers will be considered, namely, all agents whose von Neumann Morgenstern utility function is strictly concave. In order to get significant results for such a huge class of utility functions, strong conditions of positive dependence among the risks are needed.

It is definitely not enough to use some measure of dependence like the correlation coefficient. This would be possible only under very restrictive hypotheses on the choice criterion and on the parametric family of distributions for the risks. See e. g. Luciano and Kast (2001) who used value at risk and normally distributed risks, or Barrieu and El Karoui (2002, 2003), who dealt with climatic options in a normal framework.

The necessity to go beyond correlation in finance and insurance models has been emphasized among others by Embrechts et al. (1999, 2002).

The concept of dependence that will be applied is "stochastic increasingness" (or "stochastic decreasingness"). This concept was introduced under the name "positive regression dependence" by Lehmann (1966) and has been widely used in the applied probability literature (see e. g. Barlow and Proschan (1975)).

A random variable $X_{2}$ is stochastically increasing in another random variable $X_{1}$ if conditioning on higher values of $X_{1}$ the conditional distribution of $X_{2}$ becomes larger in the sense of first degree stochastic dominance.

A simple extreme case of stochastic increasingness is given by $X_{1}$ and $X_{2}$ comonotone.

Another simple case where stochastic increasingness arises is when a common environmental variable $\Theta$ is present. Assume e. g. that, given $\Theta$, the random variables $X_{1}$ and $X_{2}$ are conditionally i. i. d. normally distributed with expectation $\Theta$. If the prior distribution 
for $\Theta$ is itself normal, then $X_{2}$ is stochastically increasing in $X_{1}$.

Stochastic increasingness is an asymmetric concept, namely, the fact that $X_{2}$ is stochastically increasing in $X_{1}$ does not imply that $X_{1}$ is stochastically increasing in $X_{2}$. Nevertheless stochastic increasingness is implied by "affiliation" and implies "association" (see Karlin and Rinott (1980) and Esary et al. (1967)). Both affiliation and association are symmetric concepts of positive dependence, whose definition will be given in the following section. Affiliation is a strong concept of positive dependence which has been extensively used in economics, especially in auction theory (see Milgrom and Weber (1982)).

Call $X_{1}$ the insurable risk and $X_{2}$ the uninsurable one. If the total loss $X_{1}+X_{2}$ is stochastically increasing in $X_{1}$, then the indemnity will be proved to be nondecreasing. If $X_{2}$ is stochastically decreasing in $X_{1}$, then the identity minus the indemnity is nondecreasing. The difference with respect to the independent case is that in the dependent case the optimal indemnity is not necessarily continuous. In the model considered in this paper the revenue of the insured is a function of the sum of the losses but more general revenue functions may be considered.

With the above concepts of dependence among risks results can be obtained for all strictly risk averse expected utility maximizers. With no surprise many of these results hold true for a larger class of utility functions, those which respect second order stochastic dominance.

The paper is organized as follows: Section 2 introduces the model and analyzes the case of independent risks. Section 3 contains the general supermodularity tools that provide the qualitative properties of the efficient contracts in the cases of dependent risks. In Sections 4, 5 , and 6 properties of the efficient contracts are determined under different stochastic dependence hypotheses of the risks. Section 7 looks at robustness results in the independent case. Section 8 deals with a nonexpected utility model. Section 9 considers various side issues. All the proofs appear in Section 10.

\section{The model}

Let $(\Omega, \mathcal{A}, \mathbb{P})$ be a probability space on which all the random variables will be defined. An agent with nonrandom endowment $w$ faces two random losses $X_{1}$ and $X_{2}$. The insurance market provides insurance only for $X_{1}$, a continuous random variable with support $[0, \bar{x}]$. A feasible insurance contract is characterized by a nonnegative premium $P$ and an indemnity schedule $I:[0, \bar{x}] \rightarrow \mathbb{R}$ which satisfies $0 \leq I \leq \mathrm{Id}$ for all $x \in[0, \bar{x}]$, where Id denotes the identity function $\operatorname{Id}(x)=x$. When the insured buys the contract, she is endowed with the random wealth $W=w-P-X_{1}-X_{2}+I\left(X_{1}\right)$. The insured is assumed to have von NeumannMorgenstern preferences over random wealth represented by $\mathbb{E}[U(W)]$ where $U: \mathbb{R} \rightarrow \mathbb{R}$ is increasing, strictly concave, and $C^{1}$. Let $\hat{u}(P, I)=\mathbb{E}\left[U\left(w-X_{1}-X_{2}+I\left(X_{1}\right)-P\right)\right]$ be the indirect utility of a contract for the insured. The function $\hat{u}$ is concave, with $\hat{u}(\cdot, I)$ and $\hat{u}(P, \cdot)$ strictly concave.

By selling the contract, the insurer gets $P$ and promises to pay $I\left(X_{1}\right)$ if a loss $X_{1}$ occurs. Her profit is assumed here to be of the form $\mathbb{E}\left[V\left(P-c\left(I\left(X_{1}\right)\right)\right]\right.$ where $V: \mathbb{R} \rightarrow \mathbb{R}$ with $V(0)=0$ is increasing, strictly concave, $C^{1}$, and $c: \mathbb{R}_{+} \rightarrow \mathbb{R}_{+}$is a convex, increasing, $C^{1}$ cost function satisfying $c(0)=0$ and $c^{\prime}(0) \geq 1$. Let $\hat{v}(P, I)=\mathbb{E}\left[V\left(P-c\left(I\left(X_{1}\right)\right)\right)\right]$ be the 
indirect utility of a contract for the insurer. The function $\hat{v}$ is concave, and strictly concave if $V$ and $c$ are strictly concave.

A feasible contract $(\widetilde{P}, \tilde{I})$ (strictly) dominates $(P, I)$ if $\hat{u}(\widetilde{P}, \widetilde{I}) \geq \hat{u}(P, I)$ and $\hat{v}(\widetilde{P}, \widetilde{I}) \geq$ $\hat{v}(P, I)$ (with a strict inequality for one of the two agents). A feasible contract $(P, I)$ is Paretoefficient if it is not strictly dominated. As is well-known, a contract $(P, I)$ is Pareto-efficient if it is a solution of the following program

$$
\left(\mathcal{P}_{\alpha}\right)\left\{\begin{array}{l}
\sup _{P, I} \mathbb{E}\left[U\left(w-X_{1}-X_{2}+I\left(X_{1}\right)-P\right)\right] \text { s. t. } \\
0 \leq I \leq \mathrm{Id} \\
\mathbb{E}\left[V\left(P-c\left(I\left(X_{1}\right)\right)\right)\right] \geq \alpha, \quad \text { for some } \alpha \geq V(0)
\end{array}\right.
$$

Special attention will be paid to the case $\left(\mathcal{P}_{0}\right)$. In this case the efficient contract is individually rational.

If $\left(I^{*}, P^{*}\right)$ is an efficient contract, then there exists a multiplier $\lambda \in \mathbb{R}_{+} \operatorname{such}$ that $\left(I^{*}, P^{*}\right)$ is the solution of

$$
\max _{0 \leq I(x) \leq x, P \geq 0} \mathbb{E}\left[U\left(w-X_{1}-X_{2}+I\left(X_{1}\right)-P\right)\right]+\lambda \mathbb{E}\left[V\left(P-c\left(I\left(X_{1}\right)\right)\right)\right]
$$

Since

$$
\mathbb{E}\left[U\left(w-X_{1}-X_{2}+I\left(X_{1}\right)-P\right)\right]=\mathbb{E}\left[\mathbb{E}\left[U\left(w-X_{1}-X_{2}+I\left(X_{1}\right)-P\right) \mid X_{1}\right]\right]
$$

at the optimal premium $P^{*}$, for every $x, I^{*}(x)$ is the solution of a state by state maximization problem

$$
\max _{0 \leq I(x) \leq x} \mathbb{E}\left[U\left(w-x-X_{2}+I(x)-P^{*}\right) \mid X_{1}=x\right]+\lambda V\left(P^{*}-c(I(x))\right), \quad \text { for all } x \in[0, \bar{x}]
$$

Some dependence concepts are recalled in the following definition (see e. g. Lehmann (1966) or Joe (1997)).

Definition 2.1. (i) The random variable $X_{2}$ is stochastically increasing in $X_{1}$, denoted $X_{2} \uparrow_{\text {st }} X_{1}$ (resp strictly stochastically increasing in $X_{1}$, denoted $X_{2} \uparrow_{\text {sst }} X_{1}$ ) if the map $x \mapsto \mathbb{E}\left[f\left(X_{2}\right) \mid X_{1}=x\right]$ is nondecreasing (resp increasing) for every $f$ nondecreasing (resp increasing), for which expectations exist.

(ii) $X_{2}$ is stochastically decreasing in $X_{1}$, denoted $X_{2} \downarrow_{\mathrm{st}} X_{1}$ (resp strictly stochastically decreasing in $X_{1}$, denoted $\left.X_{2} \downarrow_{\text {sst }} X_{1}\right)$ if the map $x \mapsto \mathbb{E}\left[f\left(X_{2}\right) \mid X_{1}=x\right]$ is nonincreasing (resp decreasing) for every $f$ nondecreasing (resp increasing), for which expectations exist.

Sklar (1959) showed that any joint distribution function can be decomposed into its marginals and a dependence structure, called copula. The copula is invariant with respect to increasing transformations of the marginal components. If $X_{2} \uparrow_{\text {st }} X_{1}, Y_{1}=f_{1}\left(X_{1}\right)$, and $Y_{2}=f_{2}\left(X_{2}\right)$, with $f_{1}, f_{2}$ increasing, then $Y_{2} \uparrow_{\text {st }} Y_{1}$. This implies that stochastic increasingness 
depends on the joint distribution of $\left(X_{1}, X_{2}\right)$ only through its copula. An extreme case of stochastic increasingness is given by comonotonicity. If $X_{1}, X_{2}$ are comonotone, then, for $x<y$, all the points in the support of $X_{2} \mid X_{1}=x$ are smaller than the points in the support of $X_{2} \mid X_{1}=y$. Hence $X_{2} \uparrow_{\text {st }} X_{1}$.

Two random variables $X_{1}$ and $X_{2}$ are called affiliated if the logarithm of their joint density is supermodular (see Definition 3.1 below). They are called associated if $\operatorname{Cov}\left[f\left(X_{1}, X_{2}\right), g\left(X_{1}, X_{2}\right)\right] \geq$ 0 for any nondecreasing functions $f, g: \mathbb{R}^{2} \rightarrow \mathbb{R}$.

As mentioned in the Introduction, stochastic increasingness is a positive dependence concept that is stronger than affiliation and weaker than association.

The following terminology will be used.

Definition 2.2. (i) A contract $(P, I)$ is called a deductible contract if for some $a \in[0, \bar{x}]$ it satisfies $I(x)=(x-a)^{+}$, where $y^{+}=\max (y, 0)$.

(ii) A contract $(P, I)$ is called a generalized deductible contract if for some $a \in[0, \bar{x}]$ it satisfies

$$
I(x)=0 \quad \text { for } x \in[0, a],
$$

and the functions $I$ and $\mathrm{Id}-I$ are nondecreasing on $[a, \bar{x}]$.

(iii) A contract $(P, I)$ is called a coinsurance contract if it satisfies

$$
0<I(x)<x \quad \text { for } x \in[0, \bar{x}]
$$

and the functions $I$ and $I d-I$ are nondecreasing.

(iv) A contract $(P, I)$ is called a disappearing deductible if $I$ is nondecreasing, and there exists $a \in[0, \bar{x}]$ such that $I(x)=0$ for $x \in[0, a]$ and $I-\operatorname{Id}$ is nondecreasing on $[a, \bar{x}]$.

Since $0 \leq I \leq \mathrm{Id}$, a disappearing deductible may contain full insurance on an interval $[b, \bar{x}]$. Even if the above definitions are given in terms of contracts, notice that all their properties depend only on the indemnity.

Our benchmark case will be the case of independent risks in which the optimal contract has the same properties as in the no-background-risk case, as the following theorem shows.

Theorem 2.3. Assume that $X_{1}$ and $X_{2}$ are independent. There exist efficient contracts and any efficient contract $\left(P^{*}, I^{*}\right)$ is such that $I^{*}$ and $\mathrm{Id}-I^{*}$ are nondecreasing.

In the case $\alpha=0$ the efficient contract $\left(P^{*}, I^{*}\right)$ has the following properties.

(a) There is insurance iff

$$
c^{\prime}(0)<\frac{\left.\mathbb{E}\left[U^{\prime}\left(w-\bar{x}-X_{2}\right)\right]\right)}{\mathbb{E}\left[U^{\prime}\left(w-X_{1}-X_{2}\right)\right]}
$$

If $c^{\prime}(x)>1$ for all $\left.\left.x \in\right] 0, \bar{x}\right]$, then $\left(P^{*}, I^{*}\right)$ is a generalized deductible.

(b) If $V(x)=a x, c(x)=(1+m) x$, and (2.3) holds, then $\left(P^{*}, I^{*}\right)$ is a deductible.

(c) If furthermore $m=0$, then $\left(P^{*}, I^{*}\right)$ is full insurance. 
(d) If $V$ is strictly concave, and $c(x)=x$, then $\left(P^{*}, I^{*}\right)$ is a coinsurance contract.

When there is no background risk, or the background risk is independent, then efficient contracts are such that

(i) Agents' wealths are comonotone. Therefore anything that violates comonotonicity is ruled out: for example no insurance followed by coinsurance then full insurance violates the comonotonicity of $I$ and $\mathrm{Id}-I$. Thus only one of the following is possible:

- coinsurance,

- full insurance,

- no insurance followed by coinsurance,

- full insurance followed by coinsurance.

(ii) When there are no costs, either there is full insurance if the insurer is risk neutral, or coinsurance if she is risk averse.

(iii) When there are costs, there is a deductible (with full insurance above the deductible if the insurer is risk neutral, or coinsurance if she is risk averse).

(iv) Properties stated in Theorem 2.3 hold true for every strictly concave utility function. Stronger properties of the efficient contracts, that are induced by additional hypotheses on the utility function (e. g. HARA), do not survive the presence of an independent background risk.

\section{Supermodularity and efficient contracts in the de- pendent case}

This section contains some general results on rearrangements and supermodularity, that are at the core of all our theorems concerning the risk sharing with dependent risks. For supermodularity the reader is referred e. g. to Milgrom and Shannon (1994), Topkis (1998), Athey (2002).

First recall two definitions.

Definition 3.1. A function $\phi: \mathbb{R}^{2} \rightarrow \mathbb{R}$ is called supermodular if

$$
\phi\left(x_{1}, y_{1}\right)+\phi\left(x_{2}, y_{2}\right) \geq \phi\left(x_{1}, y_{2}\right)+\phi\left(x_{2}, y_{1}\right),
$$

for all $x_{1}<x_{2}, y_{1}<y_{2}$. If the inequality is strict, the function $\phi$ is called strictly supermodular.

A function $\phi \in C^{2}$ is supermodular iff $\partial^{2} \phi\left(x_{1}, x_{2}\right) / \partial x_{1} \partial x_{2} \geq 0$ for all $\left(x_{1}, x_{2}\right) \in \mathbb{R}^{2}$. If $\partial^{2} \phi\left(x_{1}, x_{2}\right) / \partial x_{1} \partial x_{2}>0$ for all $\left(x_{1}, x_{2}\right) \in \mathbb{R}^{2}$, then $\phi$ is strictly supermodular. 
Example 3.2. The following are examples of supermodular functions.

If $f: \mathbb{R} \rightarrow \mathbb{R}$ and $g: \mathbb{R} \rightarrow \mathbb{R}$ are either both increasing or both decreasing, and $\phi\left(x_{1}, x_{2}\right)=f\left(x_{1}\right) g\left(x_{2}\right)$, then $\phi$ is supermodular.

Consider a convex function $\psi: \mathbb{R} \rightarrow \mathbb{R}$, and define $\phi\left(x_{1}, x_{2}\right)=\psi\left(x_{1}+x_{2}\right)$. Then $\phi$ is supermodular.

Analogously, if $\xi: \mathbb{R} \rightarrow \mathbb{R}$ is concave, and $\phi\left(x_{1}, x_{2}\right)=\xi\left(x_{1}-x_{2}\right)$, then $\phi$ is supermodular. This case will be used extensively in our applications.

Definition 3.3. A function $\widetilde{I}$ is a rearrangement of $I$ with respect to $X$ if $\widetilde{I}(X)$ and $I(X)$ have the same distribution.

The following lemma generalizes some inequalities due to Hardy and Littlewood, (see Hardy et al. (1988)).

Lemma 3.4. Let $X$ be a bounded random variable with a continuous distribution $F_{X}$. Let $\widetilde{I}$ be a nondecreasing rearrangement of $I$ with respect to $X$. Then for any supermodular function $\phi$

$$
\mathbb{E}[\phi(\widetilde{I}(X), X)] \geq \mathbb{E}[\phi(I(X), X)] .
$$

If $\phi$ is strictly supermodular, then equality holds only if $I=\widetilde{I}$ a. e..

(b) For every Borel subset $A$ of $[0, \bar{x}]$

$$
\mathbb{E}\left[\mathbf{1}_{A}(X) \phi\left(\widetilde{I}_{A}(X), X\right)\right] \geq \mathbb{E}\left[\mathbf{1}_{A}(X) \phi(I(X), X)\right],
$$

where $\widetilde{I}_{A}$ is a nondecreasing rearrangement of $I$ on $A$.

A special case of Lemma 3.4 (a) goes back to Lorentz (1953). It also follows from more general inequalities for expectations of distributions with fixed marginals. See e. g. Cambanis et al. (1976) Tchen (1980). Part (b) was proved by means of different techniques by Carlier and Dana (2005).

The next two lemmata are proved using Lemma 3.4 and the supermodularity of the function $\psi_{P}(x, y)=\mathbb{E}\left[U\left(w-X_{2}-x+y-P\right) \mid X_{1}=x\right]$ when $X_{1}+X_{2} \uparrow_{\mathrm{st}} X_{1}$, and of the function $\phi_{P}(x, z)=\mathbb{E}\left[U\left(W-z-P-X_{2}\right) \mid X_{1}=x\right]$ when $X_{2} \downarrow_{s t} X_{1}$.

Lemma 3.5. Let $X_{1}+X_{2} \uparrow_{\text {st }} X_{1}$, and let $\widetilde{I}$ be a nondecreasing rearrangement of I with respect to $X_{1}$. Then for $U: \mathbb{R} \rightarrow \mathbb{R}$ concave increasing and for all $w \geq 0$ and $P \geq 0$

$$
\mathbb{E}\left[U\left(w-X_{1}-X_{2}+\widetilde{I}\left(X_{1}\right)-P\right)\right] \geq \mathbb{E}\left[U\left(w-X_{1}-X_{2}+I\left(X_{1}\right)-P\right)\right] .
$$

Moreover if $X_{1}+X_{2} \uparrow_{\text {sst }} X_{1}$, and $U$ is strictly concave, then the inequality is strict unless $I$ is nondecreasing. 
Lemma 3.6. Assume that $X_{2} \downarrow_{\text {st }} X_{1}$.

Let $Z:[0, \bar{x}] \rightarrow \mathbb{R}$ and $\widetilde{Z}$ be its nondecreasing rearrangement with respect to $X_{1}$. Then for $U: \mathbb{R} \rightarrow \mathbb{R}$ concave increasing and for all $w \geq 0$ and $P \geq 0$

$$
\mathbb{E}\left[U\left(w-X_{2}-\widetilde{Z}\left(X_{1}\right)-P\right)\right] \geq \mathbb{E}\left[U\left(w-X_{2}-Z\left(X_{1}\right)-P\right)\right]
$$

Moreover if $U$ is strictly concave and $X_{2} \downarrow_{\text {sst }} X_{1}$, then the inequality is strict unless $Z$ is nondecreasing.

The following proposition shows that, if $X_{1}+X_{2} \uparrow_{\mathrm{st}} X_{1}$, attention may be restricted to nondecreasing contracts, whereas, if $X_{2} \downarrow_{\text {st }} X_{1}$, attention may be restricted to contracts $I$ such that $\mathrm{Id}-I$ is nondecreasing. This result is the fundamental tool for the proof of Theorem 3.8, the main result of this section.

Proposition 3.7. (a) Let $X_{1}+X_{2} \uparrow_{\text {st }} X_{1}$ and $\widetilde{I}$ be a nondecreasing rearrangement of $I$ with respect to $X_{1}$. Then $(P, \widetilde{I})$ dominates $(P, I)$ (strictly if $I$ is not nondecreasing and $\left.X_{1}+X_{2} \uparrow_{\text {sst }} X_{1}\right)$.

(b) Let $X_{2} \downarrow_{\mathrm{st}} X_{1}\left(X_{2} \downarrow_{\mathrm{sst}} X_{1}\right)$ and let $Z=\mathrm{Id}-I$ and $\widetilde{Z}$ be a nondecreasing rearrangement of $Z$ with respect to $X_{1}$. Then $(P, \mathrm{Id}-\widetilde{Z})$ dominates $(P, I)$ (strictly if $Z$ is not nondecreasing and $X_{2} \downarrow_{\text {sst }} X_{1}$ or $c$ is strictly convex or $V$ is strictly concave).

The following theorem provides monotonicity properties of efficient contracts under different dependence assumptions on the risks.

Theorem 3.8. (a) Let $X_{1}+X_{2} \uparrow_{\mathrm{st}} X_{1}$. Then there exist efficient contracts, and any efficient contract is nondecreasing.

(b) Let $X_{2} \downarrow_{\text {st }} X_{1}$. Then there exist efficient contracts, and any efficient contract $I^{*}$ is such that $\mathrm{Id}-I^{*}$ is nondecreasing.

\section{The case $X_{2} \uparrow_{\text {st }} X_{1}$}

In this section $X_{2} \uparrow_{\text {st }} X_{1}$. This assumption is justified e. g. in one of the following circumstances.

An individual may insure her house, but not her car, against fire. If the car is parked in the driveway of the house and for some reason it gets on fire, there is a positive probability that the fire spreads to the house and damages it, or vice versa. Therefore, if $X_{1}$ is the insured risk related to the fire damage of the house, and $X_{2}$ is the uninsured risk related to the fire damage of the car, the assumption $X_{2} \uparrow_{\mathrm{st}} X_{1}$ is quite reasonable.

Consider a household with two individuals: one of them has health insurance, the other doesn't. Since health insurances policies cover, among other things, risks related to infectious diseases, it is reasonable to assume that two people living in the same environment, are prone to get sick at the same time, and therefore there is positive dependence between the risks related to the health of the two individuals. 
Again consider a household with two people working in the same field: one of them is insured against job loss, the other is not. Since firing of workers often depends on the general situation of the economy or of an industry, more than on their performances, it is quite likely that the two spouses could be dismissed at approximately the same time, so that the risks related to the dismissal of one is positively dependent on the risk related to the dismissal of the other.

Risks that are somehow dependent on common environmental circumstances tend to exhibit positive dependence of the form $X_{2} \uparrow_{\text {st }} X_{1}$. For instance, given $\Theta$, let $X_{1}, X_{2}$ be conditionally i. i. d. normally distributed random variables with expectation $\Theta$. If $\Theta$ itself has a normal distribution, then $X_{2}$ is stochastically increasing in $X_{1}$. Since conditional increasingness is invariant with respect to increasing transformations of the marginals, any pair of random variables that has a normal copula with positive correlation coefficient will have this property.

Lemma 4.1. If $X_{2}$ is stochastically increasing in $X_{1}$, and $X_{1}$ is nondegenerate, then $X_{1}+X_{2}$ is strictly stochastically increasing in $X_{1}$.

The following is a general existence and monotonicity result obtained by supermodularity methods.

Theorem 4.2. Assume that $X_{2} \uparrow_{\mathrm{st}} X_{1}$. Then there exist efficient contracts, and any efficient contract is nondecreasing.

The proposition that follows specifies conditions for the agent to optimally choose to have insurance in the case $\alpha=0$.

Proposition 4.3. Assume $X_{2} \uparrow_{\mathrm{st}} X_{1}$, and $\alpha=0$. Then there is no insurance iff

$$
c^{\prime}(0) \geq \frac{\mathbb{E}\left[U^{\prime}\left(w-\bar{x}-X_{2}\right) \mid X_{1}=\bar{x}\right]}{\mathbb{E}\left[U^{\prime}\left(w-X_{1}-X_{2}\right)\right]} .
$$

When there is positively dependent background risk, agents' wealths are not comonotone. Hence there may be more than two insurance regimes. The following scenario is possible: there is no insurance for small values of the insurable risk, there is full insurance for intermediate values, and partial insurance for high values of the risk.

In the case of a risk neutral insurer, then there are at most three regimes: no insurance followed by partial insurance and then by full insurance, as the following proposition shows.

Proposition 4.4. Assume that $X_{2} \uparrow_{\mathrm{st}} X_{1}$, and let $\alpha=0, V(x)=a x, c(x)=(1+m) x$, with $m \geq 0$. The following properties hold.

(a) There is no insurance iff

$$
(1+m) \geq \frac{\left.\mathbb{E}\left[U^{\prime}\left(w-\bar{x}-X_{2}\right) \mid X_{1}=\bar{x}\right]\right)}{\mathbb{E}\left[U^{\prime}\left(w-X_{1}-X_{2}\right)\right]}
$$

(b) If either $m>0$, or $X_{2} \uparrow_{\text {sst }} X_{1}$, then full insurance is not efficient. 
(c) When the efficient contract is interior, the function $\mathrm{Id}-I^{*}$ is nonincreasing.

(d) The efficient contract is a disappearing deductible.

Proposition 4.4 shows that costs do not explain deductibles anymore. In particular, if the insurer is risk neutral, there are no costs, and $X_{2} \uparrow_{\text {sst }} X_{1}$, then full insurance is not efficient, and there is a disappearing deductible. As mentioned before, the optimal contract is not necessarily continuous.

\section{The case $X_{1}+X_{2} \uparrow_{\mathrm{st}} X_{1}$ and $X_{2} \downarrow_{\mathrm{st}} X_{1}$}

In this section the two risks are negatively dependent but that their sum is positively dependent on $X_{1}$. This assumption is reasonable e. g. in the following circumstances.

Most of the insurance contracts do not cover losses due to acts of terrorism or riots. Consider then a contract that covers the loss of a car, except if the loss is due to an act of terrorism. The risk of losing the car is decomposed into an insured component $X_{1}$ and an uninsured component $X_{2}$. Since it's impossible to lose a car for two different reasons at the same time, the two components are negatively dependent in a very strong sense: whenever one is positive, the other is zero, hence they are anticomonotone, which implies $\left(X_{2} \downarrow_{s t} X_{1}\right)$. On the other hand, since the probability of losing a car due to an act of terror is in general extremely small compared to the probability of losing it due to other reasons, the total risk $X_{1}+X_{2}$ will be more heavily dependent on $X_{1}$ than on $X_{2}$, and therefore the assumption $X_{1}+X_{2} \uparrow_{\text {st }} X_{1}$ makes sense.

A person faces the risk of losing her spouse, who has life insurance. Life insurance policies usually do not cover suicide. Therefore the risk due to the loss of the spouse is decomposed into the insured component $X_{1}$ and the uninsured component $X_{2}$ relative to death due to suicide. Since the spouse cannot die due to suicide and to another reason at the same time, it follows that $X_{2} \downarrow_{\mathrm{st}} X_{1}$, but since suicide is statistically a very unlikely cause of death $X_{1}+X_{2} \uparrow_{\mathrm{st}} X_{1}$.

In general this assumption is reasonable in the following situation. Mutually exclusive causes may each give rise to some risk, but the insurance coverage is provided only for some of the causes. The probability of the claim happening for the uninsured causes is small compared to the probability of its happening for the insured causes.

Notice that, although stochastic increasingness of $X_{2}$ with respect to $X_{1}$ depends only on the copula of $\left(X_{1}, X_{2}\right)$, the hypothesis $X_{1}+X_{2} \uparrow_{\text {st }} X_{1}$ depends on the copula of $\left(X_{1}, X_{1}+X_{2}\right)$, not on the copula of $\left(X_{1}, X_{2}\right)$.

Theorem 5.1. Assume that $X_{1}+X_{2} \uparrow_{\mathrm{st}} X_{1}$ and $X_{2} \downarrow_{\mathrm{st}} X_{1}$. Then there exist efficient contracts, and any efficient contract is such that $I^{*}$ and $\mathrm{Id}-I^{*}$ are nondecreasing. Hence $I^{*}$ is 1-Lipschitz.

The continuity of the contract marks a big difference between this case and the ones considered in Sections 4 and 6. In this case, an optimal contract will be shown to have standard features: when there are costs, it is a generalized deductible. The "linear" properties 
of optimal contracts in the independent case are lost here. In particular in the case of linear costs the deductible becomes a generalized deductible.

Let $\left(P^{*}, I^{*}\right)$ be an efficient contract when $\alpha=0$.

Proposition 5.2. Assume $X_{1}+X_{2} \uparrow_{\text {st }} X_{1}$ and $X_{2} \downarrow_{\text {st }} X_{1}$. Let $\alpha=0$. The following properties hold.

(a) There is no insurance iff

$$
c^{\prime}(0) \geq \frac{\left.\mathbb{E}\left[U^{\prime}\left(w-\bar{x}-X_{2}\right) \mid X_{1}=\bar{x}\right]\right)}{\mathbb{E}\left[U^{\prime}\left(w-X_{1}-X_{2}\right)\right]},
$$

(b) If $c^{\prime}(x)>1$ for $\left.\left.x \in\right] 0, \bar{x}\right]$, then $\left(P^{*}, I^{*}\right)$ is a generalized deductible.

The following proposition deals with a risk neutral insurer with linear costs..

Proposition 5.3. Assume that $X_{1}+X_{2} \uparrow_{\mathrm{st}} X_{1}$ and $X_{2} \downarrow_{\mathrm{sst}} X_{1}$. If $\alpha=0, V(x)=$ ax , and $c(x)=(1+m) x$, then $\left(P^{*}, I^{*}\right)$ is not a deductible.

Then consider the case of no costs.

Proposition 5.4. Assume $X_{1}+X_{2} \uparrow_{\text {sst }} X_{1}$ and $X_{2} \downarrow_{\text {st }} X_{1}$. Let $\alpha=0$, and $c(x)=x$. The following properties hold.

(a) There is insurance.

(b) If either $V$ is strictly concave or if $X_{2} \downarrow_{\text {sst }} X_{1}$, then $\left(P^{*}, I^{*}\right)$ verifies $0<I^{*}(x)<x$, and is therefore a coinsurance contract.

As mentioned at the beginning, this section models the case where the background risk is small with respect to the insurable risk. It is not surprising that efficient contracts have properties which are similar to those of the benchmark model. Agents' wealths are comonotone. Costs explain deductibles (see Propositions 5.2 and 5.3). Only the linear properties of efficient contracts are not robust to such perturbations.

\section{The case $X_{1}+X_{2} \downarrow_{\text {st }} X_{1}$}

The assumption of this section $X_{1}+X_{2} \downarrow_{\text {st }} X_{1}$ is reasonable e. g. in the following circumstances.

Consider a person whose spouse buys an insurance that covers loss of life due to a plane crash, but does not have any other form of life insurance. This case resembles the one examined in the previous section, in that the insured and uninsured risk are negatively dependent, since they are due to mutually exclusive causes. Therefore if $X_{2}$ is the uninsured risk and $X_{1}$ is the insured risk related to the plane-crash death of the spouse, then $X_{2} \downarrow_{\text {st }} X_{1}$.

But now the insured risk is related to an event of very small probability compared to the 
probability of the event that corresponds to the uninsured risk. Therefore $X_{1}$ is somehow negligible with respect to $X_{2}$, and it can happen that $X_{1}+X_{2} \downarrow_{\text {st }} X_{1}$.

The case described in this section is also the case where the wealth $w$ of the insured is random, positively dependent on the insurable risk $X_{1}$ and such that $w-X_{1}$ is positively dependent on $X_{1}$.

Lemma 6.1. If $X_{1}+X_{2}$ is stochastically decreasing in $X_{1}$, and $X_{1}$ and $X_{2}$ are nondegenerate, then $X_{2}$ is strictly stochastically decreasing in $X_{1}$.

The following result is general.

Theorem 6.2. Assume that $X_{1}+X_{2} \downarrow_{\text {st }} X_{1}$. Then there exist efficient contracts, and any efficient contract is such that $\mathrm{Id}-I^{*}$ is nondecreasing, and $I^{*}$ is nonincreasing whenever interior. Hence, there exist $x_{0}, x_{1}$ with $0 \leq x_{0} \leq x_{1} \leq \bar{x}$ such that $I^{*}=\operatorname{Id}$ on $\left[0, x_{0}\right], I^{*}$ is nonincreasing on $\left[x_{0}, x_{1}\right]$, and $I^{*}=0$ on $\left[x_{1}, \bar{x}\right]$.

As in the case examined in Section 4, efficient contracts are not necessarily continuous. Theorem 6.2 does not rule out no insurance or full insurance. This is the reason why, in each specific case, these boundary solutions have to be ruled out.

Proposition 6.3. Assume that $X_{1}+X_{2} \downarrow_{\text {st }} X_{1}$. Let $\alpha=0$. The following properties hold.

(a) There is no insurance iff

$$
c^{\prime}(0) \geq \frac{\mathbb{E}\left[U^{\prime}\left(w-X_{2}\right) \mid X_{1}=0\right]}{\mathbb{E}\left[U^{\prime}\left(w-X_{1}-X_{2}\right)\right]} .
$$

(b) if $c^{\prime}(x)>1$ for all $\left.\left.x \in\right] 0, \bar{x}\right]$, full insurance is not optimal.

Again consider the case of a risk neutral insurer with no costs.

Proposition 6.4. Assume that $X_{1}+X_{2} \downarrow_{\text {sst }} X_{1}$, that $\alpha=0, V(x)=a x$, and $c(x)=x$. Then neither no insurance, nor full insurance are optimal.

\section{Robustness of linear properties}

When a risk-averse agent exchanges risk $X_{1}$ with a risk-neutral insurer who bears no cost and makes no profit, the optimal contract is full insurance. The result holds true when there is an uninsurable background risk $X_{2}$ independent of $X_{1}$. Conversely the following holds.

Proposition 7.1. Let $U$, strictly concave, increasing, and $C^{1}$, be a fixed von NeumannMorgenstern utility function. Assume that the solution to the program

$$
\left(\mathcal{P}_{\star}\right)\left\{\begin{array}{l}
\max _{(P, I)} \mathbb{E}\left[U\left(w-X_{1}-X_{2}+\left(I\left(X_{1}\right)-P\right)\right)\right] \text { s. } t . \\
0 \leq I \leq \mathrm{Id}, \\
P=\mathbb{E}\left[I\left(X_{1}\right)\right] .
\end{array}\right.
$$

is full insurance for any marginal distributions of $X_{1}$ and $X_{2}$. Then $X_{1}$ and $X_{2}$ are independent. 


\section{The case of nonexpected utilities}

The model is as in the previous section except that agents are not assumed to be expected utility maximizers. In what follows, $L^{\infty}$ denotes $L^{\infty}(\Omega, \mathcal{A}, P)$.

Definition 8.1. Let $Z, Y \in L^{\infty}$. Then $Z \succeq_{2} Y$ if $\mathbb{E}[U(Z)] \geq \mathbb{E}[U(Y)]$ for any concave increasing function $U: \mathbb{R} \rightarrow \mathbb{R}$.

Furthermore $Z \succ_{2} Y$ if $\mathbb{E}[U(Z)]>\mathbb{E}[U(Y)]$ for any strictly concave increasing function $U: \mathbb{R} \rightarrow \mathbb{R}$.

Let $u: L^{\infty} \rightarrow \mathbb{R}$ and $v: L^{\infty} \rightarrow \mathbb{R}$ be the insured and insurer's utility and $c: \mathbb{R}_{+} \rightarrow \mathbb{R}_{+}$ be a cost function fulfilling the assumptions of Section 2. A contract $(P, I)$ is efficient if it is a solution of

$$
\left(\mathcal{Q}_{\alpha}\right)\left\{\begin{array}{l}
\sup _{P, I} u\left(w-X_{1}-X_{2}+I\left(X_{1}\right)-P\right) \quad \text { s. t. } \\
0 \leq I \leq \mathrm{Id}, \\
v\left(P-c\left(I\left(X_{1}\right)\right)\right) \geq \alpha, \quad \text { for some } \alpha \geq v(0)=0 .
\end{array}\right.
$$

The following assumptions on $u$ and $v$ will be required.

(A) For $h \in\{u, v\}$, if $Z_{n} \rightarrow Z$ pointwise, then $\lim \sup h\left(Z_{n}\right) \leq h(Z)$. Furthermore $\lim _{P_{n} \rightarrow P} v\left(P_{n}\right)=v(P)$.

(B) For $h \in\{u, v\}, h$ is strictly monotone, that is, for all $Y \geq 0$ a.e. $Y \neq 0$ and all $X \in L^{\infty}$, $h(X+Y)>h(X)$.

Furthermore $\lim _{P \rightarrow \infty} u(-P)=-\infty$.

(C) For $h \in\{u, v\}, X \succeq_{2} Y$ implies $h(X) \geq h(Y)$ for any $X, Y \in L^{\infty}$.

Furthermore $X \succ_{2} Y$ implies $v(X)>v(Y)$ for any $X, Y \in L^{\infty}$.

Assumption (C) is called second-order-stochastic-dominance-preserving utility. Examples of second order stochastic dominance preserving utilities may be found in Chew and Mao (1995).

Remark 8.2. It follows from (C) that if $X$ and $Y$ have same distribution, then $u(X)=u(Y)$ and $v(X)=v(Y)$.

Proposition 8.3. Let $\widetilde{I}$ be an nondecreasing rearrangement of $I$ with respect to $X_{1}$.

(a) If $u$ and $v$ satisfy $(\mathrm{C})$, and $X_{1}+X_{2} \uparrow_{\text {st }} X_{1}$, then $(P, \widetilde{I})$ dominates $(P, I)$ (strictly if $\left.X_{1}+X_{2} \uparrow_{\text {sst }} X_{1}\right)$.

(b) If $X_{2} \downarrow_{\mathrm{st}} X_{1}$, and $\widetilde{Z}$ is a nondecreasing rearrangement of $Z=\mathrm{Id}-I$ with respect to $X_{1}$, then $(P, \mathrm{Id}-\widetilde{Z})$ dominates $(P, I)$ (strictly if $Z$ is not nondecreasing and $X_{2} \downarrow_{\text {sst }} X_{1}$ ).

Theorem 8.4. Let $u$ and $v$ satisfy (A), (B), (C). Then 
(a) If $X_{2} \uparrow_{\text {st }} X_{1}$, then there exist efficient contracts, and any efficient contract $\left(P^{*}, I^{*}\right)$ is such that that $I^{*}$ is nondecreasing.

(b) If $X_{2} \downarrow_{\mathrm{st}} X_{1}$ and $X_{1}+X_{2} \uparrow_{\mathrm{st}} X_{1}$, then there exist efficient contracts $\left(P^{*}, I^{*}\right)$ such that $I^{*}$ and $\mathrm{Id}-I^{*}$ are nondecreasing. Moreover if $X_{1}+X_{2} \uparrow_{\mathrm{sst}} X_{1}$, then any efficient contract $\left(P^{*}, I^{*}\right)$ is such that $I^{*}$ and $\mathrm{Id}-I^{*}$ are nondecreasing.

(c) If $X_{1}+X_{2} \downarrow_{\text {st }} X_{1}$, then there exist efficient contracts, and any efficient contract $\left(P^{*}, I^{*}\right)$ is such that $\mathrm{Id}-I^{*}$ is nondecreasing and $I^{*}$ is nonincreasing whenever interior.

\section{$9 \quad$ Miscellanea}

\subsection{Other dependence concepts}

Other types of dependence concepts between $X_{1}$ and $X_{2}$ may be considered. The following definition will be needed.

Definition 9.1. An increase in $X_{1}$ induces $X_{2}$ to be less risky if for every $u$ concave increasing and for all $x<y$

$$
\mathbb{E}\left[u\left(X_{2}\right) \mid X_{1}=x\right] \leq \mathbb{E}\left[u\left(X_{2}\right) \mid X_{1}=y\right] .
$$

If the inequality is strict for every $u$ strictly concave increasing, then the increase in $X_{1}$ induces $X_{2}$ to be strictly less risky. The increase in $X_{1}$ induces $X_{2}$ to be more risky if the above inequality sign is reversed.

Assuming that the insured is prudent $\left(U^{\prime \prime \prime}>0\right)$, the same results as those of Sections 4, 5 , and 6 hold. The following proposition is an example of such result.

Proposition 9.2. Assume that the insured is prudent. Assume that an increase in $X_{1}$ induces $X_{2}$ to be less risky. Then any efficient contract $\left(P^{*}, I^{*}\right)$ is such that $I^{*}$ is nondecreasing.

Let the insurer be risk neutral, let costs be affine, and let $\alpha=0$. Then there is no insurance iff

$$
(1+m) \geq \frac{\left.\mathbb{E}\left[U^{\prime}\left(w-X_{2}-\bar{x}\right) \mid X_{1}=\bar{x}\right]\right)}{\mathbb{E}\left[U^{\prime}\left(w-X_{1}-X_{2}\right)\right]}
$$

If either $m>0$, or if $X_{1}$ induces $X_{2}$ to be strictly less risky, then the efficient contract is a disappearing deductible.

Gollier (1996) considered background risks $X_{2}$ with the property that, for some $a, \mathbb{E}\left[X_{2} \mid\right.$ $\left.X_{1}=x\right]=a$ for every $x$. He then defined an increase in $X_{1}$ to induce $X_{2}$ to be less risky if $x<y$ implies

$$
E\left[u\left(X_{2}\right) \mid X_{1}=x\right] \leq E\left[u\left(X_{2}\right) \mid X_{1}=y\right],
$$

for every $u$ concave. The result stated above remains true: if the insured is prudent and if the increase in $X_{1}$ induces $X_{2}$ to be less risky, then $I^{*}$ is nondecreasing. If the insurer is risk neutral and costs are affine, if there is insurance and either $m>0$, or $X_{1}$ induces $X_{2}$ to be strictly less risky, then the efficient contract is a disappearing deductible. 


\subsection{An insurer with random wealth}

Up to now the insurer's wealth was assumed to be nonrandom. Assume now that the insurer's wealth, denoted $X_{3}$, is random. Let $X_{3}+P-c(I)$ be the insurer's wealth when the contract $(I, P)$ is offered. Assume that the insurer is risk-averse. Finally assume that $X_{3}$ and $X_{1}$ are dependent (this assumption is natural in the context of reinsurance). The following proposition holds.

Proposition 9.3. Assume that $X_{1}+X_{2} \uparrow_{\mathrm{st}} X_{1}$ and $X_{3} \uparrow_{\mathrm{st}} X_{1}$ Then any efficient contract $\left(P^{*}, I^{*}\right)$ is such that $I^{*}$ is nondecreasing. If $X_{1}+X_{2} \downarrow_{\text {st }} X_{1}$ and $X_{3}-X_{1} \downarrow_{\text {st }} X_{1}$, then any efficient contract $\left(P^{*}, I^{*}\right)$ is such that $\mathrm{Id}-I^{*}$ is nondecreasing.

\subsection{Comparative statics}

Several papers have considered comparative statics issues in the presence of background risk (references can be found in Schlesinger (2000)). Most of them dealt with parametric models where there is either coinsurance or a deductible. They focussed on comparing the optimal contract in the presence of background risk with the optimal contract without background risk.

The situation becomes much more difficult when efficient contracts are considered. If the insurer is risk neutral and faces no costs, then, under the assumptions of Sections 4 and 5, without background risk there is full insurance, and with background risk there is either a disappearing deductible or a generalized deductible. These results remain true for small values of the loading factor. So the comparison between the cases with nor without background risk is not clear-cut and it depends on the value of the risk.

\subsection{Nonseparable background risk}

In this paper the case of additively separable background risk $\left(X_{1}+X_{2}\right)$ was considered. More generally it would have been possible to consider an aggregate loss of the form $f\left(X_{1}, X_{2}\right)$ as Vercammen (2001) or Mahul (2000). Defining $\tilde{\psi}_{P}(x, y)=\mathbb{E}\left[U\left(w-f\left(x, X_{2}\right)+y-P\right) \mid X_{1}=x\right]$, if $\tilde{\psi}_{P}$ is supermodular, the optimal contract is nondecreasing. Similarly if $g_{P}(x, z)=$ $\mathbb{E}\left[U\left(w-f\left(x, X_{2}\right)+x-z-P\right) \mid X_{1}=x\right]$ is supermodular, efficient contracts are such that Id $-I$ is nondecreasing. Vercammen (2001) or Mahul (2000) consider only the independent case. In that case $\tilde{\psi}_{P}$ is supermodular if $f(\cdot, y)$ is nondecreasing, and $g(\cdot, y)$ is supermodular if $\operatorname{Id}-f(\cdot, y)$ is nondecreasing. In the case of dependent risks $\tilde{\psi}_{P}$ is supermodular if $f\left(X_{1}, X_{2}\right) \uparrow_{\text {st }} X_{1}$, whereas $g_{P}$ is supermodular if $X_{1}-f\left(X_{1}, X_{2}\right) \uparrow_{\text {st }} X_{1}$.

\section{Proofs}

\section{Section 2}

Proof of Theorem 2.3. Let $\widetilde{U}: \mathbb{R} \rightarrow \mathbb{R}$ be defined by $\widetilde{U}(x)=\mathbb{E}\left[U\left(x-X_{2}\right)\right]$. Then $\widetilde{U}$ inherits the properties of $U$. Let $\left(P^{*}, I^{*}\right)$ be an efficient contract. Then it is the solution of a program 
$\left(\mathcal{P}_{\alpha}\right)$ for some $\alpha \geq 0$. Since $X_{1}$ and $X_{2}$ are independent, $\left(\mathcal{P}_{\alpha}\right)$ may be rewritten as a standard Pareto problem without background risk:

$$
\left\{\begin{array}{l}
\sup _{P, I} \mathbb{E}\left[\widetilde{U}\left(w-X_{1}+I\left(X_{1}\right)-P\right)\right] \quad \text { s. t. } \\
0 \leq I \leq \mathrm{Id} \\
\mathbb{E}\left[V\left(P-c\left(I\left(X_{1}\right)\right)\right)\right] \geq \alpha
\end{array}\right.
$$

Hence $\left(P^{*}, I^{*}\right)$ is such that $I^{*}$ and $\mathrm{Id}-I^{*}$ are nondecreasing. Consider now $\left(\mathcal{P}_{0}\right)$, and let $\left(P^{*}, I^{*}\right)$ be its optimal solution.

(a) From (2.1) and (2.2), it follows that the contract $\left(P^{*}=0, I^{*}=0\right)$ is optimal iff there exists $\lambda \geq 0$ such that

$$
\begin{aligned}
& -\mathbb{E}\left[U^{\prime}\left(w-X_{1}-X_{2}\right)\right]+\lambda V^{\prime}(0) \leq 0 \\
& \widetilde{U}^{\prime}(w-x)=\mathbb{E}\left[U^{\prime}\left(w-x-X_{2}\right)\right] \leq \lambda V^{\prime}(0) c^{\prime}(0) \text { for all } x \in[0, \bar{x}] .
\end{aligned}
$$

Hence

$$
\mathbb{E}\left[U^{\prime}\left(w-x-X_{2}\right)\right] \leq \lambda V^{\prime}(0) c^{\prime}(0) \leq \mathbb{E}\left[U^{\prime}\left(w-X_{1}-X_{2}\right)\right] c^{\prime}(0) \quad \text { for all } x \in[0, \bar{x}]
$$

and

$$
c^{\prime}(0) \geq \frac{\mathbb{E}\left[U^{\prime}\left(w-\bar{x}-X_{2}\right)\right]}{\mathbb{E}\left[U^{\prime}\left(w-X_{1}-X_{2}\right)\right]}
$$

as was to be proven.

The fact that $I^{*}$ and $\mathrm{Id}-I^{*}$ are nondecreasing is well-known. For the sake of completeness $\left(P^{*}, I^{*}\right)$ is shown to be a generalized deductible whenever $c^{\prime}(x)>1$ for all $x \in] 0,1]$. Since $I^{*}$ and $\mathrm{Id}-I^{*}$ are comonotone, there are three possible cases: full insurance followed by coinsurance, coinsurance, and generalized deductible. The first two cases are ruled out.

First a contract such that $I(x)=x$ for $x \in\left[0, x_{0}\right]$, with $0<x_{0} \leq \bar{x}$, and $0<I(x)<x$, for $x \in\left[x_{0}, \bar{x}\right]$, is shown not to be optimal. For if it were, differentiating with respect to $P$, one would obtain for some $\lambda \geq 0$

$$
\begin{aligned}
\mathbb{E}\left[U^{\prime}\left(w-X_{1}-X_{2}+I^{*}\left(X_{1}\right)-P^{*}\right)\right] & =\lambda \mathbb{E}\left[V^{\prime}\left(P^{*}-c\left(I^{*}\left(X_{1}\right)\right)\right)\right], \\
\mathbb{E}\left[U^{\prime}\left(w-x-X_{2}+I^{*}(x)-P^{*}\right)\right] & \left.=\lambda V^{\prime}\left(P^{*}-c\left(I^{*}(x)\right)\right) c^{\prime}\left(I^{*}(x)\right)\right) \\
& >\lambda V^{\prime}\left(P^{*}-c\left(I^{*}(x)\right)\right), \quad \text { for } x \in\left[x_{0}, \bar{x}\right], \\
\mathbb{E}\left[U^{\prime}\left(w-X_{2}-P^{*}\right)\right] & \geq \lambda V^{\prime}\left(P^{*}-c(x)\right) c^{\prime}(x) \\
& >\lambda V^{\prime}\left(P^{*}-c(x)\right) \quad \text { for } x \in\left[0, x_{0}\right] .
\end{aligned}
$$

Integrating over $[0, \bar{x}]$, one gets a contradiction. 
If there is coinsurance similarly for some $\lambda>0$

$$
\begin{aligned}
\mathbb{E}\left[U^{\prime}\left(w-X_{1}-X_{2}+I^{*}\left(X_{1}\right)-P^{*}\right)\right] & =\lambda \mathbb{E}\left[V^{\prime}\left(P^{*}-c\left(I^{*}\left(X_{1}\right)\right)\right)\right], \\
\mathbb{E}\left[U^{\prime}\left(w-X_{2}-x+I^{*}(x)-P^{*}\right)\right] & \left.=\lambda V^{\prime}\left(P^{*}-c\left(I^{*}(x)\right)\right) c^{\prime}\left(I^{*}(x)\right)\right) \\
& \left.\left.>\lambda V^{\prime}\left(P^{*}-c\left(I^{*}(x)\right)\right), \quad \text { for } x \in\right] 0, \bar{x}\right],
\end{aligned}
$$

which also leads to a contradiction. Hence the only possible case is that of a generalized deductible.

(b) As is well-known, if $V(x)=a x, c(x)=(1+m) x$, the solution of $\left(\mathcal{P}_{0}\right)$ is no insurance if the cost is too high and a deductible otherwise.

(c) If $V(x)=a x, c(x)=x$, and (2.3) holds, then $\left(P^{*}, I^{*}\right)$ is full insurance.

(d) Since

$$
1<\frac{\mathbb{E}\left[U^{\prime}\left(w-X_{2}-\bar{x}\right)\right.}{\mathbb{E}\left[U^{\prime}\left(w-X_{1}-X_{2}\right)\right]}
$$

there is insurance.

Next a contract such that $I(x)=x$ for $x \in\left[0, x_{0}\right]$, with $0<x_{0} \leq \bar{x}$, and $0<I(x)<x$, for $x \in\left[x_{0}, \bar{x}\right]$, is shown not to be optimal. For if it were, differentiating with respect to $P$, then one would have for some $\lambda \geq 0$

$$
\mathbb{E}\left[U^{\prime}\left(w-X_{1}-X_{2}+I^{*}\left(X_{1}\right)-P^{*}\right)\right]=\lambda \mathbb{E}\left[V^{\prime}\left(P^{*}-I^{*}\left(X_{1}\right)\right)\right]
$$

and

$$
\begin{aligned}
\mathbb{E}\left[U^{\prime}\left(w-X_{2}-x+I^{*}(x)-P^{*}\right)\right] & =\lambda V^{\prime}\left(P^{*}-I^{*}(x)\right), \quad \text { for } x \in\left[x_{0}, \bar{x}\right], \\
\mathbb{E}\left[U^{\prime}\left(w-X_{2}-P^{*}\right)\right] & \geq \lambda V^{\prime}\left(P^{*}-x\right), \quad \text { for } x \in\left[0, x_{0}\right]
\end{aligned}
$$

Integrating over $[0, \bar{x}]$,

$$
\mathbb{E}\left[U^{\prime}\left(w-X_{2}-P^{*}\right)\right]=\lambda V^{\prime}\left(P^{*}-x\right) \quad \text { for all } x \in\left[0, x_{0}\right],
$$

which is a contradiction since the function $-V^{\prime}$ is increasing. If a regime of no insurance for low values of the risk is followed by a coinsurance regime, similarly for some $\lambda>0$ and $x_{0}>0$

$$
\mathbb{E}\left[U^{\prime}\left(w-X_{2}-x-P^{*}\right)\right]=\lambda V^{\prime}\left(P^{*}\right), x \in\left[0, x_{0}\right],
$$

which leads to a contradiction since the function $-U^{\prime}$ is increasing. 


\section{Section 3}

Proof of Lemma 3.5. For fixed $(P, w)$, let $\psi_{P}(x, y)=\mathbb{E}\left[U\left(w-X_{2}-x+y-P\right) \mid X_{1}=x\right]$. Then

$$
\frac{\partial \psi_{P}}{\partial y}(x, y)=\mathbb{E}\left[U^{\prime}\left(w-X_{2}-x+y-P\right) \mid X_{1}=x\right]=\mathbb{E}\left[U^{\prime}\left(w-X_{1}-X_{2}+y-P\right) \mid X_{1}=x\right] .
$$

Since $U^{\prime}$ is decreasing and $X_{1}+X_{2} \uparrow_{\text {st }} X_{1}$, then $\partial \psi_{P}(\cdot, y) / \partial y$ is nondecreasing.

From Lemma 3.4 it follows that

$$
\begin{aligned}
\mathbb{E}\left[U\left(w-X_{1}-X_{2}+\widetilde{I}\left(X_{1}\right)-P\right)\right] & =\int_{0}^{\bar{x}} \psi_{P}(x, \widetilde{I}(x)) \mathrm{d} F_{X_{1}}(x) \\
& \geq \int_{0}^{\bar{x}} \psi_{P}(x, I(x)) \mathrm{d} F_{X_{1}}(x) \\
& =\mathbb{E}\left[U\left(w-X_{1}-X_{2}+I\left(X_{1}\right)-P\right)\right],
\end{aligned}
$$

proving the desired result. Moreover if $U^{\prime}$ is decreasing and $X_{1}+X_{2} \uparrow_{\text {sst }} X_{1}$, then, by Lemma 3.4, the inequality is strict unless $I$ is nondecreasing.

Proof of Lemma 3.6. For fixed $(w, P)$, let $\phi_{P}(x, z)=\mathbb{E}\left[U\left(w-z-P-X_{2}\right) \mid X_{1}=x\right]$. Then

$$
\frac{\partial \phi_{P}}{\partial z}(x, z)=-\mathbb{E}\left[U^{\prime}\left(w-X_{2}-z-P\right) \mid X_{1}=x\right]
$$

Since $U^{\prime}$ is nonincreasing and $X_{2} \downarrow_{\text {st }} X_{1}$, then $\partial \phi_{P}(\cdot, z) / \partial z$ is nondecreasing. From Lemma 3.4 it follows that

$$
\begin{aligned}
\mathbb{E}\left[U\left(w-X_{2}-\widetilde{Z}\left(X_{1}\right)-P\right)\right] & =\int_{0}^{\bar{x}} \phi_{P}(x, \widetilde{Z}(x)) \mathrm{d} F_{X_{1}}(x) \\
& \geq \int_{0}^{\bar{x}} \phi_{P}(x, Z(x)) \mathrm{d} F_{X_{1}}(x) \\
& =\mathbb{E}\left[U\left(w-X_{2}-Z\left(X_{1}\right)-P\right)\right] .
\end{aligned}
$$

Moreover if $U$ is strictly concave and $X_{2} \downarrow_{\text {sst }} X_{1}$, by Lemma 3.4, then the inequality is strict unless $Z$ is nondecreasing.

Proof of Proposition 3.7. Let $X_{1}+X_{2} \uparrow_{\text {st }} X_{1}$ and $\widetilde{I}$ be a nondecreasing rearrangement of $I$ with respect to $X_{1}$. Then $v(P, \widetilde{I})=v(P, I)$. By Lemma 3.5, $u(P, \widetilde{I}) \geq u(P, I)$ with a strict inequality if $X_{1}+X_{2} \uparrow_{\text {sst }} X_{1}$ and $I$ is not nondecreasing, proving part (a).

Let $X_{2} \downarrow_{\text {st }} X_{1}\left(X_{2} \downarrow_{\text {sst }} X_{1}\right)$ and let $Z=\mathrm{Id}-I$ and $\widetilde{Z}$ be a nondecreasing rearrangement of $Z$ with respect to $X_{1}$. By Lemma 3.4, since the function $h_{P}(x, z)=V(P-c(x-z))$ is supermodular (strictly supermodular if $c$ is strictly convex or $V$ is strictly concave), the following holds

$$
v(P, \operatorname{Id}-\widetilde{Z})=\mathbb{E}\left[V\left(P-c\left(X_{1}-\widetilde{Z}\left(X_{1}\right)\right)\right)\right] \geq \mathbb{E}\left[V\left(P-c\left(I\left(X_{1}\right)\right)\right)\right]=v(P, I),
$$

with a strict inequality if $Z$ is not nondecreasing and $c$ is strictly convex or $V$ is strictly concave. Furthermore, by Lemma 3.6, $u(P, \mathrm{Id}-\widetilde{Z}) \geq u(P, I)$ with a strict inequality if $X_{2} \downarrow_{\text {sst }} X_{1}$ and $\mathrm{Id}-\widetilde{Z}$ is not nondecreasing, proving part (b). 
Proof of Theorem 3.8. (a) By Proposition 3.7 attention may be restricted to nondecreasing contracts. To show existence recall that a contract is efficient iff it is an optimal solution of a program $\left(\mathcal{P}_{\alpha}\right)$ for some $\alpha \geq 0$.

To show the existence of an optimal solution of $\left(\mathcal{P}_{\alpha}\right)$, let $\left(P_{n}, I_{n}\right)$ be a maximizing sequence with $I_{n}$ nondecreasing. Since $\lim _{P \rightarrow \infty} U(-P)=-\infty$, the sequence $P_{n}$ is bounded. By Helly's theorem, the sequence $\left(P_{n}, I_{n}\right)$ has a limit point $\left(P^{*}, I^{*}\right)$, with $I_{n} \rightarrow I^{*}$ pointwise and $I^{*}$ nondecreasing. Clearly $0 \leq I^{*} \leq \mathrm{Id}$. By Lebesgue dominated convergence theorem

$$
\mathbb{E}\left[V\left(P^{*}-c\left(I^{*}\left(X_{1}\right)\right)\right)\right]=\lim _{n} \mathbb{E}\left[V\left(P_{n}-c\left(I_{n}\left(X_{1}\right)\right)\right)\right] \geq \alpha,
$$

therefore $I^{*}$ is feasible.

Furthermore, using again Lebesgue dominated convergence theorem,

$$
\mathbb{E}\left[U\left(w-X_{1}-X_{2}+I^{*}\left(X_{1}\right)-P^{*}\right)\right]=\lim _{n} \mathbb{E}\left[U\left(w-X_{1}-X_{2}+I_{n}\left(X_{1}\right)-P_{n}\right)\right],
$$

hence $\left(P^{*}, I^{*}\right)$ is optimal for $\left(\mathcal{P}_{\alpha}\right)$. Since $U$ is strictly concave, $I^{*}\left(X_{1}\right)-P^{*}$ is unique but since $I^{*}(0)=0$, then $\left(P^{*}, I^{*}\right)$ is unique for each $\alpha$.

Let $\left(P^{*}, I^{*}\right)$ be an efficient contract. Then it is the unique optimal solution to $\left(\mathcal{P}_{\alpha}\right)$ for some $\alpha$. By Proposition $3.7\left(P^{*}, I^{*}\right)$ is dominated by $\left(P^{*}, \widetilde{I}^{*}\right)$. If $I^{*} \neq \widetilde{I}^{*}$, then, since $\hat{u}(P, \cdot)$ is strictly concave, $\left(P^{*},\left(\widetilde{I}^{*}+I^{*}\right) / 2\right)$ strictly dominates $\left(P^{*}, I^{*}\right)$, which is a contradiction. Hence $I^{*}=\widetilde{I}^{*}$, proving that $I^{*}$ is nondecreasing.

(b) This part can be proved in a similar way by considering Id $-I$ instead of $I$.

\section{Section 4}

Proof of Lemma 4.1. Denote by $[X \mid A]$ a random variable that has the distribution of $X$ given $A$, and let $=_{\text {st }}$ denote equality in law. If $X_{2} \uparrow_{\text {st }} X_{1}$, then, for $x<y$

$$
\begin{aligned}
{\left[X_{1}+X_{2} \mid X_{1}=x\right] } & { }_{\text {st }}\left[x+X_{2} \mid X_{1}=x\right] \\
& \leq_{\text {sst }}\left[y+X_{2} \mid X_{1}=x\right] \\
& \leq_{\text {st }}\left[y+X_{2} \mid X_{1}=y\right] \\
& { }_{\text {st }}\left[X_{1}+X_{2} \mid X_{1}=y\right] .
\end{aligned}
$$

Proof of Theorem 4.2. This follows from Lemma 4.1 and Theorem 3.8.

Proof of Proposition 4.3. Let $\left(P^{*}, I^{*}\right)$ be the optimal solution of $\left(\mathcal{P}_{0}\right)$. Differentiating with respect to $P$ and $I,\left(P^{*}=0, I^{*}=0\right)$ is optimal iff there exists $\lambda$ such that

$$
\begin{aligned}
-\mathbb{E}\left[U^{\prime}\left(w-X_{1}-X_{2}\right)\right]+\lambda V^{\prime}(0) & \leq 0, \\
\mathbb{E}\left[U^{\prime}\left(w-X_{2}-x\right) \mid X_{1}=x\right] & \leq \lambda V^{\prime}(0) c^{\prime}(0), \quad \text { for all } x \in[0, \bar{x}] .
\end{aligned}
$$


Since $X_{2} \uparrow_{\text {st }} X_{1}$, the map $x \mapsto \mathbb{E}\left[U^{\prime}\left(w-X_{2}-x\right) \mid X_{1}=x\right]$ is increasing and (10.2) implies

$$
\frac{\mathbb{E}\left[U^{\prime}\left(w-X_{2}-\bar{x}\right) \mid X_{1}=\bar{x}\right]}{c^{\prime}(0)} \leq \lambda V^{\prime}(0) .
$$

From (10.1) it follows that if $\left(P^{*}=0, I^{*}=0\right)$ is optimal, then either

$$
\frac{\mathbb{E}\left[U^{\prime}\left(w-X_{2}-\bar{x}\right) \mid X_{1}=\bar{x}\right]}{c^{\prime}(0)} \leq \lambda V^{\prime}(0) \leq \mathbb{E}\left[U^{\prime}\left(w-X_{1}-X_{2}\right)\right],
$$

or

$$
c^{\prime}(0) \geq \frac{\mathbb{E}\left[U^{\prime}\left(w-X_{2}-\bar{x}\right) \mid X_{1}=\bar{x}\right]}{\mathbb{E}\left[U^{\prime}\left(w-X_{1}-X_{2}\right)\right]} .
$$

Conversely if (10.3) holds, let

$$
\lambda=\frac{\mathbb{E}\left[U^{\prime}\left(w-X_{2}-\bar{x}\right) \mid X_{1}=\bar{x}\right]}{V^{\prime}(0) c^{\prime}(0)} .
$$

Then

$$
\lambda V^{\prime}(0) c^{\prime}(0) \geq \mathbb{E}\left[U^{\prime}\left(w-X_{2}-x\right) \mid X_{1}=x\right], \quad \text { for all } x \in[0, \bar{x}] .
$$

Hence (10.2) follows. From (10.3) it follows that

$$
\lambda V^{\prime}(0)=\frac{\mathbb{E}\left[U^{\prime}\left(w-X_{2}-\bar{x}\right) \mid X_{1}=\bar{x}\right]}{c^{\prime}(0)} \leq \mathbb{E}\left[U^{\prime}\left(w-X_{1}-X_{2}\right)\right],
$$

hence (10.1). Thus $\left(P^{*}=0, I^{*}=0\right)$ is optimal.

Proof of Proposition 4.4. (a) This is just a corollary of Proposition 4.3.

(b) If full insurance were optimal, differentiating with respect to $P$ and $I$, then for some $\lambda \geq 0$

$$
\begin{aligned}
\mathbb{E}\left[U^{\prime}\left(w-X_{2}-\mathbb{E}\left[X_{1}\right]\right)\right] & =\lambda, \\
\mathbb{E}\left[U^{\prime}\left(w-X_{2}-\mathbb{E}\left[X_{1}\right]\right) \mid X_{1}=x\right] & \geq \lambda(1+m) \text { for almost all } x \in[0, \bar{x}] .
\end{aligned}
$$

Integration with respect to $X_{1}$ provides a contradiction, if $m>0$.

Assume now that $m=0$. Combining (10.4) and (10.5) one gets

$$
\mathbb{E}\left[U^{\prime}\left(w-X_{2}-\mathbb{E}\left[X_{1}\right]\right) \mid X_{1}=x\right]=\lambda \text { for almost all } x \in[0, \bar{x}],
$$

which is impossible if $X_{2} \uparrow_{\text {sst }} X_{1}$, since the map $x \mapsto \mathbb{E}\left[U^{\prime}\left(w-X_{2}-\mathbb{E}\left[X_{1}\right]\right) \mid X_{1}=x\right]$ is increasing.

(c) Assume that $I$ is interior. Defining $Z(x)=x-I(x)$, then for some $\lambda \geq 0$,

$$
\mathbb{E}\left[U^{\prime}\left(w-X_{2}-Z\left(X_{1}\right)-P^{*}\right) \mid X_{1}=x\right]=\lambda(1+m) .
$$

Since $x \mapsto \mathbb{E}\left[U^{\prime}\left(w-X_{2}-z-P^{*}\right) \mid X_{1}=x\right]$ is nondecreasing and $z \mapsto \mathbb{E}\left[U^{\prime}\left(w-X_{2}-\right.\right.$ $\left.\left.z-P^{*}\right) \mid X_{1}=x\right]$ is increasing, the function Id $-I$ is nonincreasing. 
(d) If $I\left(x_{0}\right)=x_{0}$ for some $x_{0}$, then $I(x)=x$ for $x \geq x_{0}$. Indeed, for some $\lambda>0$,

$$
\mathbb{E}\left[U^{\prime}\left(w-X_{2}-\mathbb{E}\left[X_{1}\right]\right) \mid X_{1}=x_{0}\right] \geq \lambda(1+m) .
$$

Since $X_{2} \uparrow_{\text {st }} X_{1}$,

$$
\mathbb{E}\left[U^{\prime}\left(w-X_{2}-\mathbb{E}\left[X_{1}\right]\right) \mid X_{1}=x\right] \geq \lambda(1+m), \quad \text { for } x \geq x_{0} .
$$

hence $I(x)=x$ for $x \geq x_{0}$.

Now the optimal contract for $\left(\mathcal{P}_{0}\right)$ is characterized. By Theorem $4.2, I^{*}$ is nondecreasing. The optimal contract cannot be interior in a neighborhood of zero, since from (c), when interior, it must be nonincreasing. If $I^{*}(x)=x$ for $x \in\left[0, x_{0}\right]$, then as remarked above, the optimal contract would be full insurance, contradicting (b). Hence there is a regime of no insurance for low values of the risk. Then either $I^{*}$ is interior and $\mathrm{Id}-I^{*}$ is nonincreasing, or, as remarked, if $I\left(x_{0}\right)=x_{0}$ for some $x_{0}$, then $I(x)=x$ for $x \geq x_{0}$. Hence there is a disappearing deductible.

\section{Section 5}

Proof of Theorem 5.1. This follows directly from Theorem 3.8.

Proof of Proposition 5.2. Let $\left(P^{*}, I^{*}\right)$ be the optimal solution of $\left(\mathcal{P}_{0}\right)$.

(a) This part, which is similar to the previous proofs, is skipped.

(b) Since $\left(P^{*}=0, I^{*}=0\right)$ is not optimal, then $P^{*}>0$ and $I^{*} \neq 0$ (if not the constraint $\mathbb{E}\left[v\left(P^{*}-\mathbb{E}\left[c\left(I^{*}(X)\right)\right]\right)\right]=0$ would not be binding $)$.

The contract $(P, I)$ such that $I(x)=x$ for $x \in\left[0, x_{0}\right]$ with $0<x_{0} \leq \bar{x}$ and $0<I(x)<x$ for $x \in\left[x_{0}, \bar{x}\right]$ is shown not to be optimal. For if it were,

$$
\begin{aligned}
\mathbb{E}\left[U^{\prime}\left(w-X_{1}-X_{2}+I^{*}\left(X_{1}\right)-P^{*}\right)\right] & =\lambda \mathbb{E}\left[V^{\prime}\left(P^{*}-c\left(I^{*}\left(X_{1}\right)\right)\right)\right] \\
\mathbb{E}\left[U^{\prime}\left(w-X_{2}-x+I(x)-P^{*}\right) \mid X_{1}=x\right] & =\lambda V^{\prime}\left(P^{*}-c(I(x)) c^{\prime}(I(x))\right. \\
& >\lambda V^{\prime}\left(P^{*}-c(I(x)) \quad \text { for } x \in\left[x_{0}, \bar{x}\right],\right.
\end{aligned}
$$

and

$$
\begin{aligned}
\mathbb{E}\left[U^{\prime}\left(w-X_{2}-P^{*}\right) \mid X_{1}=x\right] & \geq \lambda V^{\prime}\left(P^{*}-c(x)\right) c^{\prime}(x) \\
& >\lambda V^{\prime}\left(P^{*}-c(x)\right) \text { for } x \in\left[0, x_{0}\right] .
\end{aligned}
$$

Integration over $[0, \bar{x}]$, provides a contradiction with (10.6).

The contract $(P, I)$ such that $0<I(x)<x$ for $x \in[0, \bar{x}]$ is not optimal, since $\mathbb{E}\left[U^{\prime}\left(w-X_{2}-x+I(x)-P^{*}\right) \mid X_{1}=x\right]=\lambda V^{\prime}\left(P^{*}-c(I(x)) c^{\prime}(I(x)), \quad\right.$ for all $x \in[0, \bar{x}]$ also contradicts (10.6) since $c^{\prime}(I(x))>1$. Hence there exists $x_{0}>0$, such that $I^{*}(x)=$ 0 for all $x \in\left[0, x_{0}\right]$, and $0<I^{*}(x)<x$ for $x>x_{0}$. 
Proof of Proposition 5.3. When interior, I fulfills the equation

$$
\mathbb{E}\left[U^{\prime}\left(w-X_{2}-x+I(x)-P^{*}\right) \mid X_{1}=x\right]=\lambda(1+m) .
$$

If $X_{2} \downarrow_{\text {sst }} X_{1}$, then $x \mapsto \mathbb{E}\left[U^{\prime}\left(w-X_{2}-z-P^{*}\right) \mid X_{1}=x\right]$ is decreasing, $z \mapsto \mathbb{E}\left[U^{\prime}(w-\right.$ $\left.\left.X_{2}-z-P^{*}\right) \mid X_{1}=x\right]$ is increasing, hence Id $-I$ is increasing, which rules out the existence of a deductible.

Proof of Proposition 5.4. (a) Since

$$
1<\frac{\mathbb{E}\left[U^{\prime}\left(w-X_{2}-\bar{x}\right) \mid X_{1}=\bar{x}\right]}{\mathbb{E}\left[U^{\prime}\left(w-X_{1}-X_{2}\right)\right]},
$$

as in the previous cases, one can verify that $\left(P^{*}=0, I^{*}=0\right)$ is not optimal.

(b) The contract $(P, I)$ such that $I(x)=x$ for $x \in\left[0, x_{0}\right]$ with $0<x_{0} \leq \bar{x}$, and $0<I(x)<x$ for $x \in\left[x_{0}, \bar{x}\right]$ is shown not to be optimal if $V$ is strictly concave or if $X_{2} \downarrow_{\text {sst }} X_{1}$. For if it were, then

$$
\begin{aligned}
\mathbb{E}\left[U^{\prime}\left(w-X_{2}-x+I^{*}(x)-P^{*}\right)\right] & =\lambda \mathbb{E}\left[V^{\prime}\left(P^{*}-I^{*}(x)\right)\right], \\
\mathbb{E}\left[U^{\prime}\left(w-X_{2}-P^{*}\right) \mid X_{1}=x\right] & \geq \lambda V^{\prime}\left(P^{*}-x\right), \quad \text { for } x \in\left[0, x_{0}\right], \\
\mathbb{E}\left[U^{\prime}\left(w-X_{2}-x+I^{*}(x)-P^{*}\right) \mid X_{1}=x\right] & =\lambda V^{\prime}\left(P^{*}-I^{*}(x)\right), \quad \text { for } x \in\left[x_{0}, \bar{x}\right] .
\end{aligned}
$$

Since $\mathbb{E}\left[U^{\prime}\left(w-X_{2}-P^{*}\right) \mid X_{1}=x_{0}\right]=\lambda V^{\prime}\left(P^{*}-x_{0}\right)$, under either of the above hypotheses, $\mathbb{E}\left[U^{\prime}\left(w-X_{2}-P^{*}\right) \mid X_{1}=x\right]>\lambda V^{\prime}\left(P^{*}-x\right)$ for $x \in\left[0, x_{0}\right]$ contradicting (10.7).

Similarly it is not possible to have $I(x)=0$ for $x \in\left[0, x_{0}\right]$ with $0<x_{0} \leq \bar{x}$ and $0<I(x)<x$ for $x \in\left[x_{0}, \bar{x}\right]$ if either $V$ is strictly concave or if $X_{1}+X_{2} \uparrow_{\text {sst }} X_{1}$. Hence $0<I^{*}(x)<x$.

\section{Section 6}

Proof of Lemma 6.1. Let $x<y$. Then

$$
\left[X_{1}+X_{2} \mid X_{1}=x\right] \geq_{\text {st }}\left[X_{1}+X_{2} \mid X_{1}=y\right]
$$

is equivalent to

$$
\left[x+X_{2} \mid X_{1}=x\right] \geq_{\text {st }}\left[y+X_{2} \mid X_{1}=y\right],
$$

which in turn is equivalent to

$$
\left[X_{2} \mid X_{1}=x\right] \geq_{\text {st }}\left[y-x+X_{2} \mid X_{1}=y\right] .
$$

Since

$$
\left[y-x+X_{2} \mid X_{1}=y\right] \geq_{\text {sst }}\left[X_{2} \mid X_{1}=y\right],
$$

transitivity gives the result. 
Proof of Theorem 6.2. This follows from Lemma 6.1 and Theorem 3.8.

Proof of Proposition 6.3. Let $\left(P^{*}, I^{*}\right)$ be the optimal solution of $\left(\mathcal{P}_{0}\right)$.

(a) The proof is analogous to that of Proposition 4.3, using the fact that the map $x \mapsto$ $\mathbb{E}\left[U^{\prime}\left(w-X_{2}-x \mid X_{1}=x\right)\right]$ is nonincreasing.

(b) If full insurance were optimal, then

$$
\begin{aligned}
\mathbb{E}\left[U^{\prime}\left(w-X_{2}-\mathbb{E}\left[X_{1}\right]\right)\right] & =\lambda \mathbb{E}\left[V^{\prime}\left(\mathbb{E}\left[X_{1}\right]-c\left(X_{1}\right)\right)\right] \\
\mathbb{E}\left[U^{\prime}\left(w-X_{2}-\mathbb{E}\left[X_{1}\right]\right) \mid X_{1}=x\right] & \geq \lambda V^{\prime}\left(\mathbb{E}\left[X_{1}\right]-c(x)\right) c^{\prime}(x) \\
& >\lambda V^{\prime}\left(\mathbb{E}\left[X_{1}\right]-c(x)\right) \quad \text { for all } x \in[0, \bar{x}]
\end{aligned}
$$

Integration with respect to $X_{1}$, gives a contradiction.

Proof of Proposition 6.4. The contract $\left(P^{*}=0, I^{*}=0\right)$ is optimal iff there exists $\lambda \geq 0$ such that

$$
\begin{aligned}
-\mathbb{E}\left[U^{\prime}\left(w-X_{1}-X_{2}\right)\right]+\lambda & \leq 0 \\
\mathbb{E}\left[U^{\prime}\left(w-X_{2}-x\right) \mid X_{1}=x\right] & \leq \lambda \quad \text { for all } x \in[0, \bar{x}]
\end{aligned}
$$

Since, $X_{1}+X_{2} \downarrow_{\text {sst }} X_{1}, x \mapsto \mathbb{E}\left[U^{\prime}\left(w-X_{2}-x\right) \mid X_{1}=x\right]$ is decreasing and (10.8) and (10.9) are equivalent to

$$
\mathbb{E}\left[U^{\prime}\left(w-X_{2}\right) \mid X_{1}=0\right] \leq \lambda \leq \mathbb{E}\left[U^{\prime}\left(w-X_{1}-X_{2}\right)\right]
$$

there is a contradiction.

Let's now prove that full insurance is not optimal. If it were, differentiating with respect to $P$ and $I$, one would have for some $\lambda \geq 0$

$$
\begin{array}{r}
\mathbb{E}\left[U^{\prime}\left(w-X_{2}-\mathbb{E}\left[X_{1}\right]-P^{*}\right)\right]=\lambda \\
\mathbb{E}\left[U^{\prime}\left(w-X_{2}-\mathbb{E}\left[X_{1}\right]-P^{*}\right) \mid X_{1}=x\right] \geq \lambda \quad \text { for almost all } x \in[0, \bar{x}],
\end{array}
$$

hence

$$
\mathbb{E}\left[U^{\prime}\left(w-X_{2}-\mathbb{E}\left[X_{1}\right]-P^{*}\right) \mid X_{1}=x\right]=\lambda \quad \text { for almost all } x \in[0, \bar{x}] .
$$

Since $X_{2} \downarrow_{\text {sst }} X_{1}$, the map $x \mapsto \mathbb{E}\left[U^{\prime}\left(w-X_{2}-\mathbb{E}\left[X_{1}\right]-P^{*}\right) \mid X_{1}=x\right]$ is decreasing and there is a contradiction. 


\section{Section 7}

Proof of Proposition 7.1. If full insurance is the solution to the problem $\left(\mathcal{P}_{\star}\right)$, differentiating with respect to $P$ and $I$, then there exists $\lambda \geq 0$ such that

$$
\begin{array}{r}
\mathbb{E}\left[U^{\prime}\left(-X_{2}-\mathbb{E}\left[X_{1}\right]\right)=\lambda,\right. \\
\mathbb{E}\left[U^{\prime}\left(-X_{2}-\mathbb{E}\left[X_{1}\right]\right) \mid X_{1}=x\right] \geq \lambda, \quad \text { for all } x \in[0, \bar{x}] .
\end{array}
$$

Hence $\mathbb{E}\left[U^{\prime}\left(-X_{2}-\mathbb{E}\left[X_{1}\right]\right) \mid X_{1}=x\right]=\lambda$ for all $x$. Therefore $\operatorname{Cov}\left[U^{\prime}\left(-X_{2}-\mathbb{E}\left[X_{1}\right]\right), X_{1}\right]=$ 0 for any marginal distribution of $X_{1}$ and $X_{2}$. Call $Z=U^{\prime}\left(X_{2}-\mathbb{E}\left[X_{1}\right]\right)$. Then $\operatorname{Cov}\left[Z, X_{1}\right]=0$ for all marginal distributions of $Z$ and $X_{1}$. Then by Lehmann (1966)

$$
\begin{aligned}
\operatorname{Cov}\left[Z, X_{1}\right] & =\int_{-\infty}^{\infty} \int_{-\infty}^{\infty}\left[F_{Z, X_{1}}(s, t)-F_{Z}(s) F_{X_{1}}(t)\right] \mathrm{d} s \mathrm{~d} t \\
& =\int_{0}^{1} \int_{0}^{1}\left[C_{Z, X_{1}}(u, v)-u v\right] \mathrm{d} F_{Z}^{-1}(u) \mathrm{d} F_{X_{1}}^{-1}(v) \\
& =0 \text { for all } F_{Z}, F_{X_{1}} .
\end{aligned}
$$

where $C_{Z, X_{1}}$ is the copula of $F_{Z, X_{1}}$. This is possible only if $C_{Z, X_{1}}(u, v)=u v$, namely, if $Z$ and $X_{1}$ are independent, which in turns implies that $X_{1}$ and $X_{2}$ are independent.

\section{Section 8}

Proof of Proposition 8.3. This is a corollary of Proposition 3.7.

Proof of Theorem 8.4. (a) Assume that $X_{2} \uparrow_{\text {st }} X_{1}$. By Lemma 4.1 and Proposition 8.3 attention may be restricted to nondecreasing contracts. Any efficient contract is a solution of $\left(\mathcal{Q}_{\alpha}\right)$ for some $\alpha \geq 0$.

To show the existence of a solution of $\left(\mathcal{Q}_{\alpha}\right)$, let $\left(P_{n}, I_{n}\right)$ be a maximizing sequence with $I_{n}$ nondecreasing. Since $\lim _{P \rightarrow \infty} u(-P)=-\infty$, the sequence $P_{n}$ is bounded. By Helly's theorem, the sequence $\left(P_{n}, I_{n}\right)$ has a limit point $\left(P^{*}, I^{*}\right)$, with $I_{n} \rightarrow I^{*}$ pointwise and $I^{*}$ nondecreasing. Clearly $0 \leq I^{*} \leq \mathrm{Id}$. Since $v$ fulfills $(\mathrm{A})$, then

$$
v\left(P^{*}-c\left(I^{*}\right)\right) \geq \lim \sup v\left(P_{n}-c\left(I_{n}\right)\right) \geq \alpha,
$$

therefore $v\left(P^{*}-c\left(I^{*}\right)\right) \geq \alpha$.

Since $u$ fulfills $(\mathrm{A})$, then

$$
u\left(w-X_{1}-X_{2}+I^{*}\left(X_{1}\right)-P^{*}\right) \geq \lim \sup u\left(w-X_{1}-X_{2}+I_{n}\left(X_{1}\right)-P_{n}\right),
$$

hence $\left(P^{*}, I^{*}\right)$ is optimal with $I^{*}$ is nondecreasing, which proves the existence of a nondecreasing optimal solution of $\left(\mathcal{Q}_{\alpha}\right)$.

To prove that any efficient contract $\left(P^{*}, I^{*}\right)$ is such that $I^{*}$ is nondecreasing, let $\alpha$ be such that $\left(P^{*}, I^{*}\right)$ is the optimal solution of $\left(\mathcal{Q}_{\alpha}\right)$. By Lemma 4.1 , since $X_{2} \uparrow_{\text {st }} X_{1}$, 
then $X_{1}+X_{2} \uparrow_{\text {sst }} X_{1}$, hence by Lemma 3.5, if $I^{*}$ is not nondecreasing, then $w-X_{1}-$ $X_{2}+\widetilde{I}^{*}\left(X_{1}\right)-P^{*} \succ_{2} w-X_{1}-X_{2}+I^{*}\left(X_{1}\right)-P^{*}$, hence $u\left(w-X_{1}-X_{2}+\widetilde{I}^{*}\left(X_{1}\right)-P^{*}\right)>$ $u\left(w-X_{1}-X_{2}+I^{*}\left(X_{1}\right)-P^{*}\right)$, whereas $v\left(P^{*}-c\left(\widetilde{I}^{*}\right)\right)=v\left(P^{*}-c\left(I^{*}\right)\right)$, a contradiction. Therefore, any efficient contract $\left(P^{*}, I^{*}\right)$ is such that $I^{*}$ is nondecreasing.

(b) To prove the existence of efficient contracts, by Proposition 8.3 attention may be restricted to contracts $\left(P^{*}, I^{*}\right)$ such that both $I^{*}$ and $\mathrm{Id}-I^{*}$ are nondecreasing. The style of the proof will be as in part (a). If $X_{1}+X_{2} \uparrow_{\text {sst }} X_{1}$, then, by Lemma 3.5, any optimal solution $\left(P^{*}, I^{*}\right)$ is such that $I^{*}$ is nondecreasing. To show that $\mathrm{Id}-I^{*}$ is nondecreasing, let $Z^{*}=\mathrm{Id}-I^{*}$ and $\widetilde{Z}^{*}$ be its nondecreasing rearrangement with respect to $X_{1}$. Since $X_{2} \downarrow_{\text {st }} X_{1}$, by Lemma 3.6, for any $U$ concave

$$
\mathbb{E}\left[U\left(w-X_{2}-\widetilde{Z}^{*}\left(X_{1}\right)-P^{*}\right)\right] \geq \mathbb{E}\left[U\left(w-X_{2}-Z^{*}\left(X_{1}\right)-P^{*}\right)\right],
$$

hence, since $u$ fulfills (A),

$$
u\left(w-X_{2}-\widetilde{Z}^{*}\left(X_{1}\right)-P^{*}\right) \geq u\left(w-X_{2}-Z^{*}\left(X_{1}\right)-P^{*}\right) .
$$

If $V$ is strictly concave, then $\widetilde{L}(x, z)=V\left(P^{*}-c(x-z)\right)$ is strictly supermodular.

By Lemma 3.4 , if $Z^{*} \neq \widetilde{Z}^{*}$, then

$$
\mathbb{E}\left[V\left(P^{*}-c\left(X_{1}-\widetilde{Z}^{*}\left(X_{1}\right)\right)\right)\right]>\mathbb{E}\left[V\left(P^{*}-c\left(X_{1}-Z^{*}\left(X_{1}\right)\right)\right)\right],
$$

for any $V$ strictly concave. Hence, since $v$ fulfills $(\mathrm{C})$,

$$
v\left(P^{*}-c\left(X_{1}-\widetilde{Z}^{*}\left(X_{1}\right)\right)\right)>v\left(P^{*}-c\left(X_{1}-Z^{*}\left(X_{1}\right)\right)\right) .
$$

Therefore, for some $\varepsilon>0$, the contract $\left(P^{*}-\varepsilon\right.$, Id $\left.-\widetilde{Z}^{*}\right)$ is feasible and dominates $\left(P^{*}, I^{*}\right)$ contradicting its optimality. Hence $Z^{*}=\widetilde{Z}^{*}$ as was to be proven.

(c) By Lemma 6.1 and Proposition 8.3 attention may be restricted to contracts $I$ such that $\mathrm{Id}-I$ is nondecreasing. Existence of an optimal contract follows from the same type of arguments as above. So does nondecreasingness of $\mathrm{Id}-I^{*}$. To show that $I^{*}$ is nonincreasing whenever interior, let $A=\left\{x: 0<I^{*}(x)<x\right\}$, and let $I=$ $I^{*}$ for every $x \in A^{c}$ and $I$ be the nonincreasing rearrangement of $I^{*}$ on $A$. Then $v\left(P^{*}-c(I)\right)=v\left(P^{*}-c\left(I^{*}\right)\right)$ since $v$ fulfills $(\mathrm{C})$. Furthermore for any concave $U$, $\mathbb{E}\left[U\left(w-X_{1}-X_{2}+I\left(X_{1}\right)-P^{*}\right)\right] \geq \mathbb{E}\left[U\left(w-X_{1}-X_{2}+I^{*}\left(X_{1}\right)-P^{*}\right)\right]$ with a strict inequality unless $I^{*}$ is nonincreasing on $A$. Hence $u\left(w-X_{1}-X_{2}+I\left(X_{1}\right)-P^{*}\right) \geq$ $u\left(w-X_{1}-X_{2}+I^{*}\left(X_{1}\right)-P^{*}\right)$ with a strict inequality unless $I^{*}$ is nonincreasing on $A$.

\section{Section 9}

Proof of Proposition 9.2. Since this proof is very tedious, only the main lines of the argument will be exposed. To show that any efficient contract $\left(P^{*}, I^{*}\right)$ is such that $I^{*}$ is nondecreasing, 
the same method of proof as in Proposition 3.7 is used. Any contract $(P, I)$ is shown to be dominated by $(P, \widetilde{I})$ with $\widetilde{I}$ a nondecreasing rearrangement of $I$ with respect to $X_{1}$.

First remark that, if $X_{1}$ induces $X_{2}$ to be less risky, then it induces $X_{1}+X_{2}$ to be strictly less risky. If $\widetilde{I}$ is a nondecreasing rearrangement of $I$ with respect to $X_{1}$, then for all concave increasing function $U: \mathbb{R} \rightarrow \mathbb{R}$ and for all $w \geq 0$ and $P \geq 0$,

$$
\mathbb{E}\left[U\left(w-X_{1}-X_{2}+\widetilde{I}\left(X_{1}\right)-P\right)\right] \geq \mathbb{E}\left[U\left(w-X_{1}-X_{2}+I\left(X_{1}\right)-P\right)\right]
$$

Indeed, as in Lemma 3.5 below, let $\psi_{P}(x, y)=\mathbb{E}\left[U\left(w-X_{2}-x+y-P\right) \mid X_{1}=x\right]$. Then

$$
\frac{\partial \psi_{P}}{\partial y}(x, y)=\mathbb{E}\left[U^{\prime}\left(w-X_{2}-x+y-P\right) \mid X_{1}=x\right]
$$

Since $-U^{\prime}$ is concave increasing, $x \rightarrow \mathbb{E}\left[U^{\prime}\left(w-X_{2}-x+y-P\right) \mid X_{1}=x\right]$ is nondecreasing and $\partial \psi_{P}(\cdot, y) / \partial y$ is nondecreasing which implies that $\psi_{P}$ is supermodular. The other assertions follow either from the fact that $x \rightarrow \mathbb{E}\left[U^{\prime}\left(w-X_{2}-x+y-P\right) \mid X_{1}=x\right]$ is nondecreasing or from the fact that $x \rightarrow \mathbb{E}\left[U^{\prime}\left(w-X_{2}-\mathbb{E}\left(X_{1}\right)\right) \mid X_{1}=x\right]$ is nondecreasing.

Proof of Proposition 9.3. The same method of proof as in Proposition 3.7 is used. Any contract $(P, I)$ is dominated by $(P, \widetilde{I})$ with $\widetilde{I}$ a nondecreasing rearrangement of $I$ with respect to $X_{1}$. In fact by Lemma 3.5

$$
\mathbb{E}\left[U\left(w-X_{1}-X_{2}+\widetilde{I}\left(X_{1}\right)-P\right)\right] \geq \mathbb{E}\left[U\left(w-X_{1}-X_{2}+I\left(X_{1}\right)-P\right)\right] .
$$

Let $h_{P}(x, y)=\mathbb{E}\left[V\left(X_{3}-y+P\right) \mid X_{1}=x\right]$. Then

$$
\frac{\partial h_{P}}{\partial y}(x, y)=\mathbb{E}\left[-V^{\prime}\left(X_{3}-y+P\right) \mid X_{1}=x\right] .
$$

Since $X_{3} \uparrow_{\text {st }} X_{1}$, the function $x \rightarrow \mathbb{E}\left[-V^{\prime}\left(X_{3}-y+P\right) \mid X_{1}=x\right]$ is nondecreasing, proving the supermodularity of $h_{P}$. By Lemma 3.4

$$
\mathbb{E}\left[V\left(X_{3}-\widetilde{I}\left(X_{1}\right)+P\right)\right] \geq \mathbb{E}\left[V\left(X_{3}-I\left(X_{1}\right)+P\right)\right],
$$

proving the desired result. The proof of the next assertion is similar.

\section{Acknowledgments}

The authors thank two referees for their useful comments and observations.

\section{References}

Arrow, K. J. (1963) Uncertainty and the welfare of medical care. Amer. Econom. Rev. 53, 941-973. 
Arrow, K. J. (1970) Essays in the Theory of Risk-Bearing. North-Holland Publishing Co., Amsterdam.

Arrow, K. J. (1974) Optimal insurance and generalized deductibles. Scand. Actuar. J. $1-42$.

Athey, S. (2002) Monotone comparative statics under uncertainty. Quart. J. Econom. 117, $187-223$.

Barlow, R. E. and Proschan, F. (1975) Statistical Theory of Reliability and Life Testing. Holt, Rinehart and Winston, Inc., New York.

Barrieu, P. and El Karoui, N. (2002) Optimal design of derivatives in illiquid markets. Quant. Finance 2, 181-188.

Barrieu, P. and El Karoui, N. (2003) Structuration optimale de produits financiers et diversification en présence de sources de risque non-négociables. C. R. Math. Acad. Sci. Paris 336, 493-498.

Borch, K. (1962) Equilibrium in a reinsurance market. Econometrica 30, 424-444.

Cambanis, S., Simons, G., and Stout, W. (1976) Inequalities for $E k(X, Y)$ when the marginals are fixed. Z. Wahrscheinlichkeitstheorie und Verw. Gebiete 36, 285-294.

Carlier, G. and Dana, R.-A. (2005) Rearrangement inequalities in non convex economic models. J. Math. Econom. To appear.

Chew, H. S. and MaO, H. M. (1995) A Schur concave characterization of risk aversion for non-expected utility preferences. J. Econom. Theory 67, 402-435.

Doherty, N. A. and Schlesinger, H. (1983a) The optimal deductible for an insurance policy when initial wealth is random. J. Bus. 56, 555-565.

Doherty, N. A. and Schlesinger, H. (1983b) Optimal insurance in incomplete markets. J. Polit. Econ. 91, 1045-1054.

Eeckhoudt, L., Gollier, C., and Schlesinger, H. (1996) Changes in background risk and risk taking behavior. Econometrica 64, 683-689.

Eeckhoudt, L. and Kimball, M. (1992) Background risk, prudence, and the demand for insurance. In Dionne, G. (ed.), Contributions to Insurance Economics, 239-254. Kluwer, Dordrecht.

Embrechts, P., McNeil, A., and Straumann, D. (1999) Correlation: Pitfalls and alternatives. A short, non-technical article. Risk Magazine 12, 69-71.

Embrechts, P., McNeil, A., and Straumann, D. (2002) Correlation and dependence in risk management: properties and pitfalls. In DempsteR, M. (ed.), Risk Management: Value at Risk and Beyond, 176-223. Cambridge University Press, Cambridge. 
Esary, J. D., Proschan, F., and Walkup, D. W. (1967) Association of random variables, with applications. Ann. Math. Stat 38, 1466-1474.

Finkelshtain, I., Kella, O., and Scarsini, M. (1999) On risk aversion with two risks. J. Math. Econom. 31, 239-250.

Franke, G., Stapleton, R. C., and Subrahmanyam, M. G. (1998) Who buys and who sells options: the role of options in an economy with background risk. J. Econom. Theory 82, 89-109.

Franke, G., Stapleton, R. C., and Subrahmanyam, M. G. (2004) Background risk and the demand for state-contingent claims. Econom. Theory 23, 321-335.

Gollier, C. (1996) Optimum insurance of approximate losses. J. Risk Insurnce 63, 369380.

Gollier, C. (2001) The Economics of Risk and Time. MIT Press, Cambridge.

Gollier, C. and Pratt, J. W. (1996) Risk vulnerability and the tempering effect of background risk. Econometrica 64, 1109-1123.

Hardy, G. H., Littlewood, J. E., and Pólya, G. (1988) Inequalities. Cambridge Mathematical Library. Cambridge University Press, Cambridge. Reprint of the 1952 edition.

JEWITT, I. (1987) Risk aversion and the choice between risky prospects: the preservation of comparative statics results. Rev. Econom. Stud. 54, 73-85.

Joe, H. (1997) Multivariate Models and Dependence Concepts. Chapman \& Hall, London.

Karlin, S. and RinotT, Y. (1980) Classes of orderings of measures and related correlation inequalities. J. Multivariate Anal. 10, 467-498.

Kinlstrom, R. E., Romer, D., and Williams, S. (1981) Risk aversion with random initial wealth. Econometrica 49, 911-920.

Kimball, M. S. (1993) Standard risk aversion. Econometrica 61, 589-611.

Lehmann, E. L. (1966) Some concepts of dependence. Ann. Math. Statist. 37, 1137-1153.

Lorentz, G. G. (1953) An inequality for rearrangements. Amer. Math. Monthly 60, 176179.

LuCiAno, E. and KASt, R. (2001) A value at risk approach to background risk. Geneva Papers Risk Insurance Theory 26, 91-115.

Mahul, O. (2000) Optimum crop insurance under joint yield and price risk. J. Risk Insurance 67, 109-122. 
Milgrom, P. and Shannon, C. (1994) Monotone comparative statics. Econometrica 62, $157-180$.

Milgrom, P. R. and Weber, R. J. (1982) A theory of auctions and competitive bidding. Econometrica 50, 1089-1122.

NaChman, D. C. (1982) Preservation of 'more risk averse' under expectations. J. Econom. Theory 28, 361-368.

Pratt, J. W. and Zeckhauser, R. J. (1987) Proper risk aversion. Econometrica 55, $143-154$.

Raviv, A. (1979) The design of an optimal insurance policy. Amer. Econ. Rev. 69, 84-96.

Ross, S. A. (1981) Some stronger measures of risk aversion in the small and the large with applications. Econometrica 49,621-638.

Schlesinger, H. (2000) The theory of insurance demand. In Dionne, G. (ed.), Handbook of Insurance, 131-151. Kluwer, Boston.

Schlesinger, H. and Doherty, N. A. (1985) Incomplete markets for insurance: An overview. J. Risk Insurance 52, 402-423.

Sklar, M. (1959) Fonctions de répartition à $n$ dimensions et leurs marges. Publ. Inst. Statist. Univ. Paris 8, 229-231.

TChen, A. H. (1980) Inequalities for distributions with given marginals. Ann. Probab. 8, 814-827.

Topkis, D. M. (1998) Supermodularity and Complementarity. Princeton University Press, Princeton, NJ.

Vercammen, J. (2001) Optimal insurance with nonseparable background risk. J. Risk Insurance 68, 437-447. 